\title{
Flattop regulates basal body docking and positioning in mono- and multiciliated cells
}

\section{Moritz Gegg 1,2, Anika Böttcher, ${ }^{1,2}$, Ingo Burtscher ${ }^{1,2}$, Stefan Hasenoeder, ${ }^{1,2}$, Claude Van Campenhout ${ }^{3}$, Michaela Aichler ${ }^{4}$, Axel Walch ${ }^{4}$, Seth G N Grant ${ }^{5,6}$, Heiko Lickert ${ }^{1,2 *}$}

${ }^{1}$ Institute of Stem Cell Research, Helmholtz Center Munich, Munich, Germany; ${ }^{2}$ Institute of Diabetes and Regeneration Research, Helmholtz Center Munich, Munich, Germany; ${ }^{3}$ Genetique du Developpement, L'Institut de biologie et de médecine moléculaires, Université libre de Bruxelles, Gosselies, Belgium; ${ }^{4}$ Research Unit Analytical Pathology, Helmholtz Center Munich, Munich, Germany; ${ }^{5}$ Centre for Clinical Brain Sciences, University of Edinburgh, Edinburgh, United Kingdom; ${ }^{6}$ Centre for Neuroregeneration, Univeristy of Edinburgh, Cambridge, United Kingdom

Abstract Planar cell polarity (PCP) regulates basal body (BB) docking and positioning during cilia formation, but the underlying mechanisms remain elusive. In this study, we investigate the uncharacterized gene Flattop (Fltp) that is transcriptionally activated during PCP acquisition in ciliated tissues. Fltp knock-out mice show BB docking and ciliogenesis defects in multiciliated lung cells. Furthermore, Fltp is necessary for kinocilium positioning in monociliated inner ear hair cells. In these cells, the core PCP molecule Dishevelled 2, the BB/spindle positioning protein Dlg3, and Fltp localize directly adjacent to the apical plasma membrane, physically interact and surround the BB at the interface of the microtubule and actin cytoskeleton. Dlg3 and Fltp knock-outs suggest that both cooperatively translate PCP cues for BB positioning in the inner ear. Taken together, the identification of novel $\mathrm{BB} /$ spindle positioning components as potential mediators of $\mathrm{PCP}$ signaling might have broader implications for other cell types, ciliary disease, and asymmetric cell division. DOI: 10.7554/eLife.03842.001

\section{Introduction}

The conserved PCP signaling pathway regulates the orientation of cells and organelles within the plane of an epithelium and is crucially important for developmental patterning as well as organ morphogenesis, homeostasis, and physiology (Seifert and Mlodzik, 2007; Wang and Nathans, 2007; Peng and Axelrod, 2012; Wallingford, 2012). Pioneering studies in Drosophila and Xenopus have revealed that global, non-cell autonomous, and cell intrinsic signaling mechanisms act in concert to establish tissue polarity. Core PCP molecules including Van Gogh-like (Vangl1-2), Cadherin EGF LAG seven-pass G-type receptor (Celsr1-3), Frizzled (Fzd3, 6), Dishevelled (Dvl1-3), and Prickle (Pk1-2) are localized asymmetrically at the cell cortex to provide polarity information for morphogenesis and oriented cell division. Significant progress has been made in understanding the asymmetric core PCP localization in vertebrates but it is less clear how this regulates cytoskeletal rearrangements that drive morphogenesis via tissue specific downstream effector molecules (Wallingford, 2012). Thus, the identification of novel PCP effectors that indicate pathway activity and mediate signaling and/or morphogenesis will be the key to unravel the function of this molecular pathway in development and disease.

Besides the Rho family of GTPases, which are also implicated in apical-basal (A-B) polarity establishment, the best-studied PCP effector molecules are Inturned (Intu) and Fuzzy (Fuz) 
eLife digest Epithelial tissues are sheets of cells that line the surface of many parts of the body, including the airways and the inner ear. Small hair-like structures called cilia can be found on the top surface of many epithelial cells and are arranged in a precise, ordered pattern. Such patterning ensures that cilia can work in a co-ordinated manner, for example by beating together to help clearing mucus from airways.

Cilia grow out from 'basal bodies' and, like many other important structures in a cell, these basal bodies must be oriented along the correct side of an epithelial tissue. This is achieved by 'planar cell polarity signaling', which makes sure that the structures inside a cell are correctly aligned, and ensures that polarized cells themselves are correctly oriented across the epithelial tissue. Disruption of this signaling can result in developmental defects.

Some proteins help to establish polarity in a cell by altering the cell's cytoskeleton-the structural support and transport network of the cell. A 'core' complex of proteins then coordinates how the cells are arranged throughout the epithelial tissue. Although many of the proteins involved in each of these roles are known, how they interact with each other to establish planar cell polarity remains poorly understood.

Now, Gegg et al. report that, in mice, a protein called Flattop functions to position basal bodies-and thus cilia-by working together with another protein called Dlg3. In mice that cannot produce Flattop, cilia formation is defective in the lung, and the cilia in the inner ear are positioned incorrectly. Gegg et al. found that in the inner ear, Flattop and Dlg3 physically interact with each other and two other proteins-including one of the core proteins involved in planar cell polarity. This protein complex then surrounds the basal bodies at the point where they connect to the cell's cytoskeleton.

Future challenges will be to clarify how the protein complex anchors to the cytoskeleton and how it interacts with other core planar cell polarity proteins in the cells of the inner ear. It will also be important to see whether this protein complex fulfills a similar role in other ciliated epithelial tissues.

DOI: 10.7554/eLife.03842.002

(Collier and Gubb, 1997; Park et al., 2006, 2008; Gray et al., 2009). Both directly regulate ciliogenesis by mediating the assembly of the apical actin cytoskeleton but are not required for the polarized accumulation of core PCP components. The core PCP molecule Dvl2 localizes near the base of cilia and functions together with Intu and Rho GTPases to dock and polarize BBs for cilia formation and directed ciliary beating (Park et al., 2008). BBs are amplified deep in the cytoplasm of multiciliated cells (MCCs) and apical plasma membrane (PM) transport depends on Dvl and the vesicle trafficking protein Sec8. Up-to-date it is not understood how core PCP molecules physically connect to effector molecules, how this leads to asymmetric membrane polarization and cytoskeletal rearrangements, and if these mechanisms are conserved among different cell types in various organs and during evolution.

First functional evidence for PCP in lung development came from the analysis of Celsr1, Vangl2, and Scribble (Scrib) mutant mice, which showed defects in branching morphogenesis and narrowed lung airways due to cytoskeletal and junctional defects (Yates et al., 2010). Multiciliated lung cells first arise at embryonic day (E) 14.0 in the trachea as well as in the main bronchi (Jain et al., 2010). Similar to the mucociliary epithelium in frog, differentiation depends on BB amplification, docking, and orientation that allows the formation of hundreds of motile cilia. The differentiation of multiciliated lung cells and the dynamics of the underlying cell biological processes can be modeled in air liquid interface (ALI) cultures of primary mouse tracheal epithelial cells (mTECs) (You et al., 2002; Vladar and Stearns, 2007; Vladar et al., 2012). Asymmetric localization of core PCP molecules at apical junctions regulates the orientation of motile cilia along the longitudinal tissue axis for directed beating and mucus clearing. This likely interdepends on non-cell autonomous cues and intrinsic polarized microtubule (MT) network topology (Vladar et al., 2012). Currently, PCP effector molecules that link core molecules, BBs, polarized MTs, and the actin cytoskeleton have not been identified. A better understanding of these molecular processes could provide further insight into a multitude of ciliary dysfunction syndromes of the lung and other organs. 
The best-established model to study PCP in vertebrates is the organ of Corti in the inner ear (IE). Mechanosensory hair cells $(\mathrm{HCs})$ are arranged in one inner $(\mathrm{IHC})$ and three outer $\mathrm{HC}(\mathrm{OHC})$ rows. The lateral polarization of the $\mathrm{V}$-shaped actin-based stereocilia bundles on $\mathrm{HCs}$ strongly depends on ciliogenesis and PCP for proper sound perception (Montcouquiol et al., 2003; Wang et al., 2005, 2006; Jones and Chen, 2008). Core PCP molecules like Celsr1, Dvl2/3, Fz3/6, and Vangl2 are localized to distinct apical membrane compartments of $\mathrm{HCs}$ and supporting cells (Ezan and Montcouquiol, 2013). This differential localization seems not sufficient to instruct morphogenesis of actin-rich hair bundles in mammals (Jones and Chen, 2008). Instead, it depends on opposing localization of evolutionarily conserved spindle positioning and apical polarity proteins that serve as a blueprint for kinocilium migration and bundle formation (Ezan et al., 2013; Tarchini et al., 2013). It remains unclear, how core PCP molecules couple to spindle positioning complexes and the actin cytoskeleton to orchestrate morphogenesis.

Spindle positioning proteins as well as the actin and MT cytoskeleton act together with cues from the cell cortex, such as apical junctions and polarity proteins to direct spindle positioning in mammalian epithelial cells (Kunda and Baum, 2009). In addition to Inscuteable (mInsc in mammals), Partner of Inscuteable (mPins/LGN in mammals) and the G-protein coupled receptor $\mathrm{Ga}_{1}$ and other evolutionary conserved proteins, such as the basolateral determinants and septate junction localized proteins Dlg and Scrib, regulate A-B spindle positioning (Knoblich, 2008; Siller and Doe, 2008). In mammals, five Dlgs have been identified and we have recently shown that their function has diverged during evolution (Van Campenhout et al., 2011). Interestingly, Dlg3 is the only family member that is involved in apical transport and required for tight junction (TJ) consolidation. Taken together, the function of Drosophila Dlg in spindle positioning (Bellaiche et al., 2001; Johnston et alo, 2009; Bergstralh et al., 2013) and mammalian Dlg3 in PCP establishment (Van Campenhout et al., 2011), suggested to us that Dlg3 could mediate PCP-dependent BB positioning.

In this study, we identified Fltp as a gene expressed in regions of active PCP signaling including the node, the MCCs of the lung, and the sensory hair cells of the inner ear. Knock-out analysis revealed that Fltp is required for BB docking and cilia formation in the lung as well as BB and kinocilium positioning in the IE. Using ALI cultures, we show that Fltp expression is induced while BBs are amplified and docked at the apical PM in differentiating MCCs. Fltp localizes next to BBs, and MT plus ends in the apical actin network and is required for efficient BB docking and cilia formation. We provide evidence that Dlg3 functions together with Fltp to position BBs and kinocilia in the inner ear. Dlg3 and Fltp physically interact with each other, the core PCP protein Dvl2, and the pericentriolar matrix protein $\mathrm{y}$-Tubulin, suggesting that we have identified a novel BB positioning complex in the inner ear. Together, our data implicate that Fltp is a novel regulator important for BB docking and positioning in mono- and multiciliated cell types that acquire PCP.

\section{Results}

\section{Identification of Fltp as a Foxa2 target gene}

We discovered Fltp as a functionally non-annotated gene (1700009P17RiK) in a screen to identify Foxa2 target genes involved in polarity establishment (Burtscher and Lickert, 2009) and expressed in monociliated node cells (Tamplin et al., 2008; Kinzel et al., 2010). The enrichment of the transcription factor FOXA2 on conserved binding sites in the promoter region of the human FLTP ortholog (C1Orf192), suggests that FLTP is a direct target of FOXA2 (Figure 1A) (Weedon et alo, 2014). The murine Fltp gene consists of six exons and the spliced mRNA codes for an open-reading frame (ORF) of 567 nucleotides that translates into a protein of 189 amino acids (Figure 1B,C). An alignment of the proteins of different species reveals a high conservation during evolution as well as an $\mathrm{N}$-terminal $\mathrm{SH} 3$ binding domain and a C-terminal proline-rich repeat (PRR) (Figure 1C). Interestingly, the mouse $1700009 P 17 R i K$ cDNA was also identified in a screen for mRNAs highly abundant in ciliated tissues (McClintock et al., 2008).

To analyze the Fltp protein in more detail, we raised two polyclonal rabbit antibodies against a central and a C-terminal epitope (Figure 1C). We confirmed the specificity of these antibodies in western blot analysis and immunocytochemistry in transiently transfected HEK293T cells (Figures 1figure supplement 1A-G, 2D). Endogenous Fltp protein localizes to the apical PM, the $B B$, and the primary cilium in monociliated mouse node cells, suggestive for a function of Fltp in BB transport, positioning, and/or ciliogenesis (Figure 2F, G). 


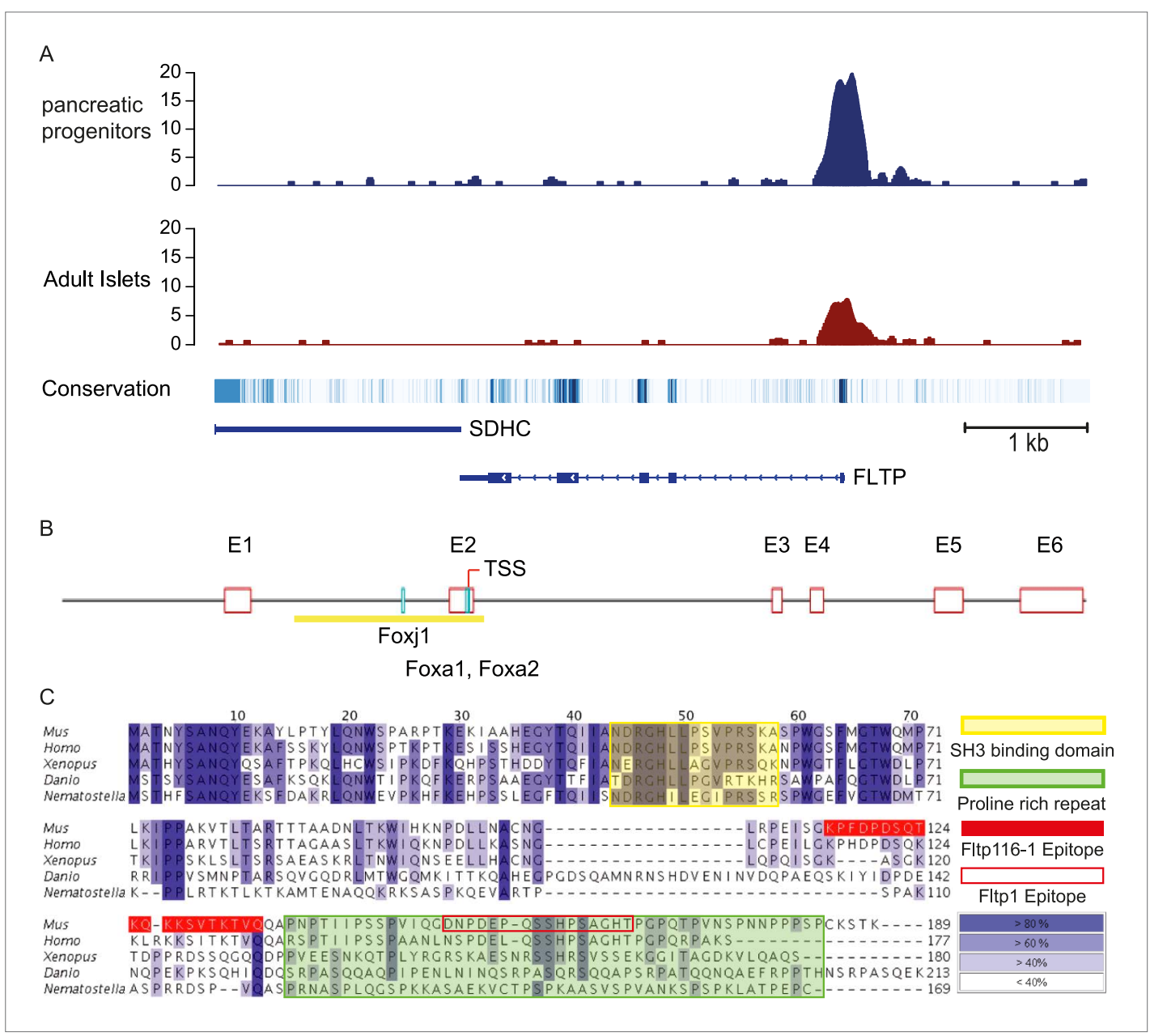

Figure 1. FLTP has active FOXA2 binding sites in its promoter and is conserved among species. (A) A high amount of the endodermal transcription factor FOXA2 binds the human FLTP promoter in pancreatic progenitors and in adult islets, indicating that FLTP is a direct target of FOXA2 and expressed in these cells. (B) Fltp shows predicted (Genomatix) Foxj1, Foxa1, and Foxa2 binding sites in its promoter (clear red boxes: exons (E1-E6); yellow box: promoter; TSS: transcriptional start site; light blue boxes: Foxj1, Foxa1, Foxa2 binding sites). (C) Fltp protein alignment shows high conservation between different species (highest conservation in the first 76 amino acids). The mouse and human proteins are highly homologous (yellow box: SH3 binding domain; green box: predicted proline rich repeat (PRR); red filled box: peptide sequence of the Fltp116-1 epitope; red empty box: peptide sequence of the Fltp1 epitope; dark blue indicates conservation over 80\%; lighter colors indicate less conservation).

DOI: 10.7554/eLife.03842.003

The following source data and figure supplements are available for figure 1:

Source data 1. Mendelian ratio of Fltp intercrosses on different backgrounds. DOI: 10.7554/eLife.03842.004

Figure supplement 1. Fltp antibody specificity.

DOI: 10.7554/eLife.03842.005

Figure supplement 2. Fltp construct and confirmation of the knock-out strategy. DOI: 10.7554/eLife.03842.006

\section{Fltp is expressed in mono- and multiciliated tissues during PCP acquisition}

To explore Fltp expression and function in vivo, we generated an Fltp ${ }^{z V}$ knock-in/knock-out allele by replacing the entire ORF by a multicistronic lacZ-Venus reporter cassette (Figure 1-figure supplement 2A and Supplemental 'Materials and methods'). PCR genotyping as well as Southern and western blotting analysis confirmed the targeted homologous recombination and the generation of a null allele (Figure 1-figure supplement 2B-D). Fltp homozygous knock-out mice are born at roughly the 

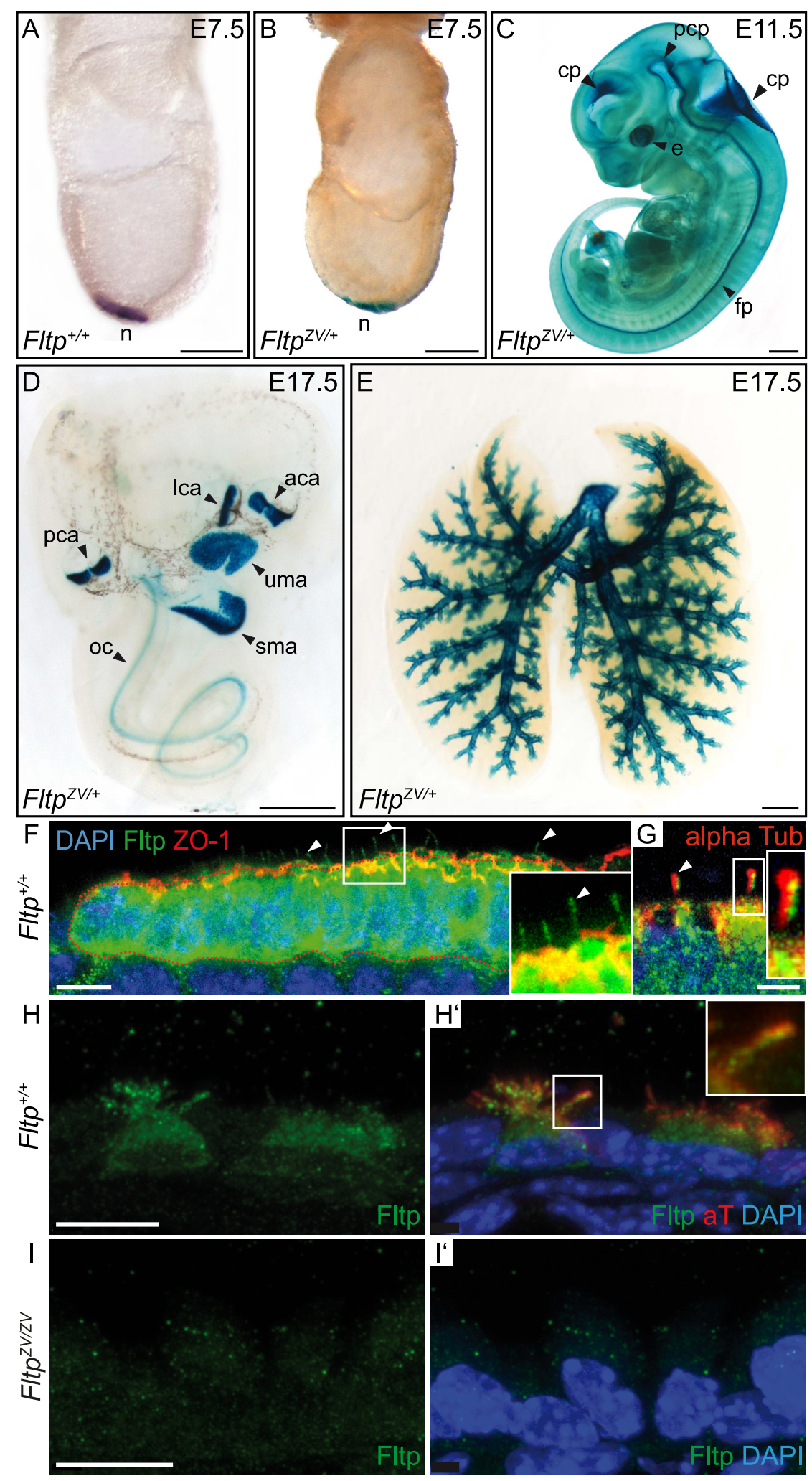

Figure 2. Fltp reporter and protein is detectable in mono- and multiciliated tissues. (A and B) mRNA (Fltp in situ hybridization) (A) and lacZ reporter expression (B) are restricted to the node (n) at E7.5. (C-E)Whole-mount lacZ stained and benzyl alcohol/benzyl benzoate (BABB) cleared Fltp ${ }^{2 V /+}$ embryo and organs. (C) E11.5 embryo reveals Figure 2. Continued on next page 
Figure 2. Continued

reporter expression in the choroid plexi (cp), prechordal plate (pcp), eye (e), and floor plate (fp). (D) E17.5 IE shows reporter expression in posterior crista ampullaris (pca), lateral crista ampullaris (lca), anterior crista ampullaris (aca), utricular macula (uma), and saccular macula (sma) of the vestibular part as well as in the organ of Corti (oc). (E) E17.5 lung shows lacZ reporter activity in multiciliated lung epithelial cells. ( $\mathbf{F}$ and $\mathbf{G}$ ) Whole-mount antibody stained embryo (node, E7.75) analyzed by LSM reveals Fltp protein in vesicular fashion in the cytoplasm and along primary cilia (white arrow heads). (H-I') Immunohistochemistry on cryosections combined with LSM analysis reveals Fltp at the apical plasma membrane and at cilia in multiciliated lung epithelial cells of Fltp ${ }^{+/+}$adult animals $\left(\mathbf{H}_{\text {and }} \mathbf{H}^{\prime}\right)$. No

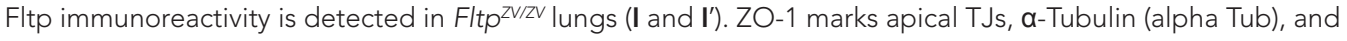
acetylated-Tubulin (aT), the tubulin network and cilia; nuclei are marked by DAPI and Fltp by Fltp1 (F and $\mathbf{G})$ and Fltp116-1 (H-I'). Scale bars; $100 \mu \mathrm{m}$ (A and B), $500 \mu \mathrm{m}$ (C, D, E), $10 \mu \mathrm{m}(\mathbf{F}), 4 \mu \mathrm{m}(\mathbf{G})$, and $10 \mu \mathrm{m}\left(\mathbf{H}-\mathbf{I}^{\prime}\right)$.

DOI: 10.7554/eLife.03842.007

The following figure supplement is available for figure 2:

Figure supplement 1. Additional Fltp reporter expression in mono- and multiciliated tissues. DOI: 10.7554/eLife.03842.008

expected Mendelian ratio and are viable and fertile on CD1, C57BL/6NCrl and 129S6/SvEvTac background (Figure 1-source data 1).

Next, we characterized the temporal and spatial expression pattern of the Fltp reporter during embryonic development. LacZ reporter activity in Fltp $\mathrm{ZV} /+$ animals is first detectable in the embryonic node at E7.5 (Figure 2B), which accurately reflects endogenous mRNA expression (Figure 2A) and confirmed our previous findings (Tamplin et al., 2008; Lange et al., 2012). At E8.5-13.5, strong reporter activity is detected in the eye, notochord, floor plate of the neural tube, and all four choroid plexi (Figure 2C, Figure 2-figure supplement 1A-D), tissues that are either mono- or multiciliated and depend on active PCP signaling. Consistent with a potential function in the PCP pathway, LacZ reporter activity in the IE coincides with BB docking and kinocilium positioning at E13.5 (Figure 2figure supplement 1E) (McKenzie et al., 2004; Ezan and Montcouquiol, 2013). The Fltp reporter remains expressed during development and early postnatal life in all six sensory regions of the IE (Figure 2D, Figure 5A, Figure 2-figure supplement 1C,E,F). Furthermore, Fltp reporter activity strongly correlates with the spatial and temporal onset of ciliogenesis in the lung trachea and the main bronchi at E14.5-17.5 (Figure 2E, Figure 2-figure supplement 1G, H). In adult lungs at postnatal day (P) 30, the dual lacZ and H2B-Venus reporter remains expressed in MCCs of the bronchioles, but not in non-ciliated cells of the alveoli (Figure 2-figure supplement $11, J)$. To confirm the reporter studies, we analyzed cell type-specific and subcellular localization of the endogenous Fltp protein in the embryonic node and adult lung epithelium. Whole-mount antibody staining combined with laser scanning microscopy (LSM) on gastrula-stage embryos revealed that the Fltp protein is localized at the apical PM, TJs, primary cilia, and in the cytoplasm of node cells (Figure 2F, G). Immunohistochemistry on lung sections further shows that Fltp is highly enriched at the apical cortex and in cilia of the multiciliated bronchial epithelial cells (Figure $2 \mathrm{H}, \mathrm{H}^{\prime}$ ). The absence of Fltp immunoreactivity in Fltp ${ }^{Z \mathrm{~V} / Z \mathrm{~V}}$ lung sections further confirmed antibody specificity (Figure 2I,I'). Taken together, Fltp reporter and endogenous expression accurately correlate with onset of PCP establishment in the embryonic node, floor plate, choroid plexi, IE, and lung, which is indicative for a function in or downstream of the PCP pathway.

\section{Fltp regulates $B B$ docking and cilia formation in multiciliated lung cells}

The embryonic and adult lung depends on PCP signaling for branching morphogenesis, BB docking, and cilia formation (Yates et al., 2010; Vladar et alı, 2012). First, we investigated the highly stereotypic branching pattern in cleared lacZ stained lungs at P60 (Metzger et al., 2008). No difference was detectable in Fltp ${ }^{T 2 A i C r e /+} ; R 26^{R /+}(\mathrm{Gt}[\mathrm{ROSA}] 26 \mathrm{Sor})$ controls lungs with normal levels of Fltp protein (Soriano, 1999; Lange et al., 2012), Fltp $\mathrm{FV}^{Z \mathrm{+}}$ heterozygous and Fltp $\mathrm{F}^{Z V / Z V}$ homozygous mutant lungs, indicating that Fltp has no function during branching morphogenesis (Figure 2E, Figure 3A-C). In contrast, measurement and quantification of terminal lung bronchiole diameters revealed a dosedependent and statistically significant constriction of the distal airways (Figure 3A-D), suggesting that normal Fltp levels are important for lung airway homeostasis. To better understand the etiology of the airway constrictions, we analyzed Fltp expression and function on cellular level in Fltp ${ }^{+/+}$, Fltp ${ }^{Z V /+}$, and Fltp ${ }^{Z V / Z V}$ adult lungs at P7 and P30 (Figure 3E-L). Fltp-driven H2B-Venus reporter activity was confined to the nuclei of multiciliated epithelial cells in Fltp ${ }^{Z V /+}$ and Fltp $2 \mathrm{~V} / Z \mathrm{~V}$ lungs at P7 and P30. LSM 


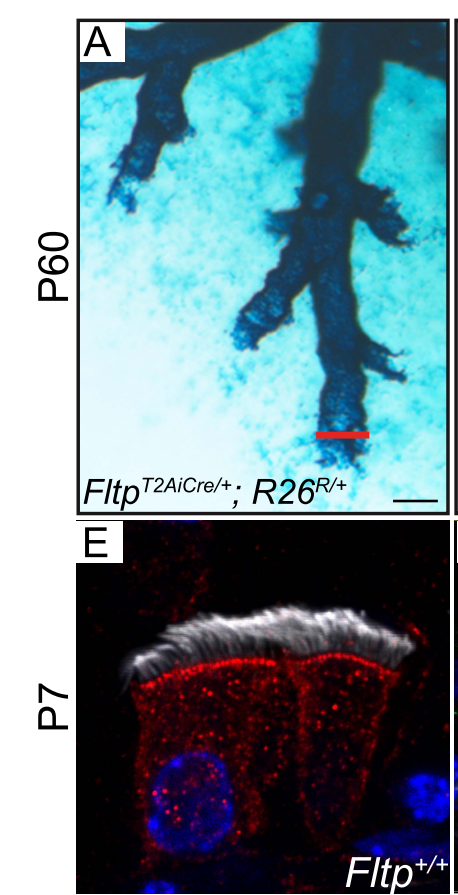

DAPI $\gamma$ Tub aT

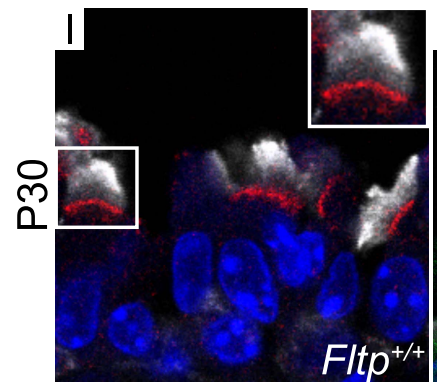

DAPI $\gamma$ Tub aT

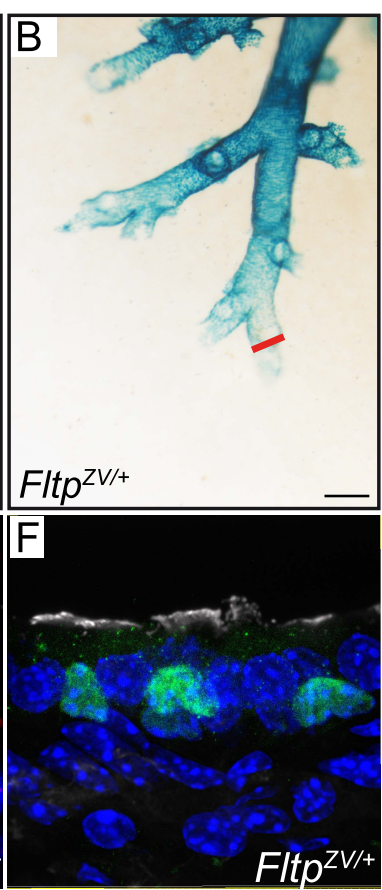

GFP aT

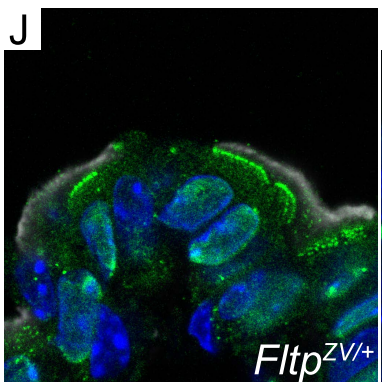

DAPI $\gamma$ Tub GFP aT
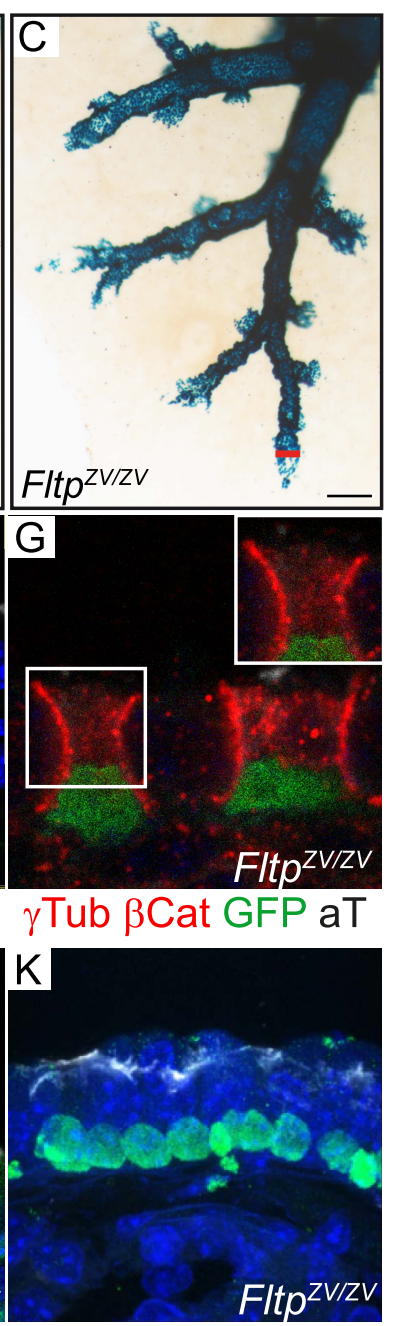

DAPI GFP aT
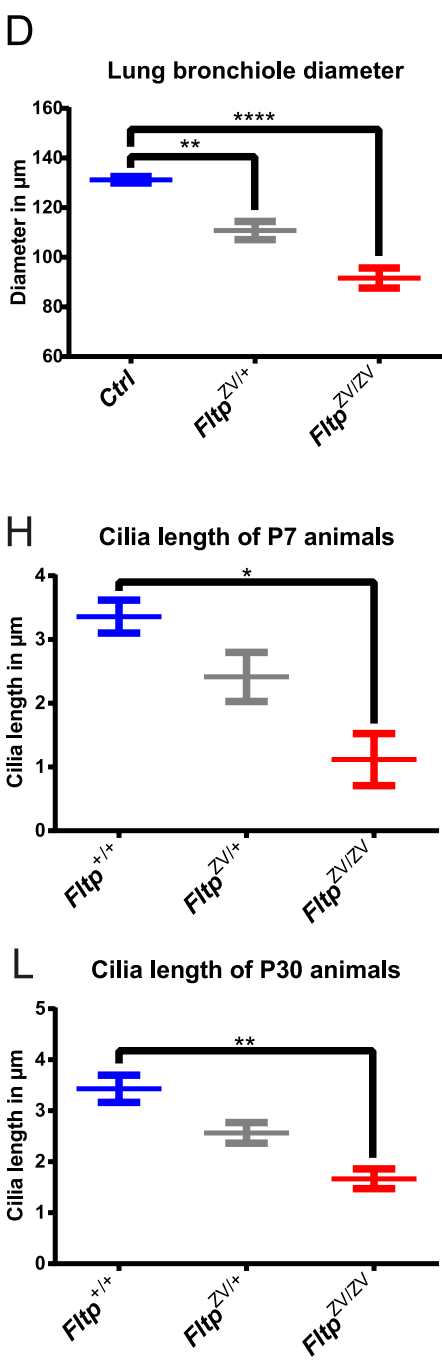

Figure 3. Loss of Fltp leads to constricted distal airways and cilia formation defects in the lung. (A-C) Whole-mount lacZ stained and BABB cleared distal airways of left lung lobes at P60. Red lines show how diameters were measured. (A) Fltp ${ }^{T 2 A i C r e /+} ; R^{2} 26^{R /+}$ is used as control (Ctrl). (B and C) Fltp ${ }^{2 \mathrm{~V} /+}$ and Fltp ${ }^{Z V / Z V}$ animals show constricted distal airways. (D) Average distal airway diameter of Ctrl animals is $132.25 \mu \mathrm{m}$ ( $\mathrm{n}=4 ; 125$ bronchi), of Fltp ${ }^{Z V /+}$ animals is $99.68 \mu \mathrm{m}(\mathrm{n}=3 ; 90)$, and of Fltp ${ }^{Z V / Z V}$ animals is $90.06 \mu \mathrm{m}(\mathrm{n}=3$; 95). ( $\mathbf{E}-\mathbf{G}$ and $\mathbf{I}-\mathbf{K})$ Immunohistochemistry on cryosections of lung distal airway epithelium combined with LSM analysis. (E and I) In Fltp ${ }^{+/+}$animals BBs project cilia at the apical surface. ( $\mathbf{F}$ and $\mathbf{J}$ ) In Fltp $\mathrm{IV}^{\mathrm{V} /+}$ animals less and shorter cilia are detectable. (G and K) Fltp ${ }^{2 V / Z V}$ animals often show absence of or shorter cilia at the apical surface. Note that only very few BBs are docked at the apical surface. $(\mathbf{H}$ and $\mathbf{L})$ Fltp ${ }^{2 V / Z V}$ animals show significant shorter cilia than Fltp ${ }^{+/+}$animals. In total we examined $\mathrm{n}=3\left(81\right.$ cells) for Fltp ${ }^{+/+}, \mathrm{n}=2(22)$ for $\mathrm{Fltp}^{2 \mathrm{~V} /+}$, and $n=2$ (93) for Fltp ${ }^{Z V / Z V}$ P7 animals. For P30 we examined $n=3$ (121 cells) for Fltp ${ }^{+/+}, n=3$ (134) for Fltp ${ }^{2 V /+}$, and $n=2$ (138) for Fltp ${ }^{2 V / Z V}$. BBs are marked by $\mathrm{Y}$-Tubulin ( $\mathrm{YTub}$ ), cilia by acetylated-Tubulin ( $\mathrm{aT})$, cell membrane by $\beta$-Catenin ( $\beta$ Cat), nuclei by DAPI, and nuclei of Fltp reporter expressing cells by GFP. Statistical analysis uses an one way ANOVA ( ${ }^{* *} p=0.0028 ;{ }^{* \star *} p<0.0001$ for $(\mathbf{D})$ and ${ }^{*} p=0.0143 ;{ }^{* \star} p=0.0025$ for $(\mathbf{H}$ and $\mathbf{L})$ ). Error bars show the 95\% confidence interval of the mean (D) and the standard error of the mean ( $\mathbf{H}$ and $\mathbf{L})$. Scale bars; $200 \mu \mathrm{m}(\mathbf{A}-\mathbf{C})$.

analysis further confirmed that cilia formation was significantly affected in a dose-dependent fashion (Figure 3E-L), similar to PCP effector knock-down phenotypes in the mucociliary epithelium of Xenopus laevis (Park et al., 2006, 2008).

To analyze the dynamic cellular and molecular requirement for Fltp during BB docking, cilia formation, and PCP establishment, we employed the mTEC culture system (You et al., 2002; Vladar et al., 2012). Switching mTEC culture to ALI conditions induces a differentiation process of lung progenitor cells towards a MCC phenotype. During differentiation the progenitor cells pass through different maturation stages (Figure 4A): Monociliated lung progenitor cells (stage I) start centrosome/BB 


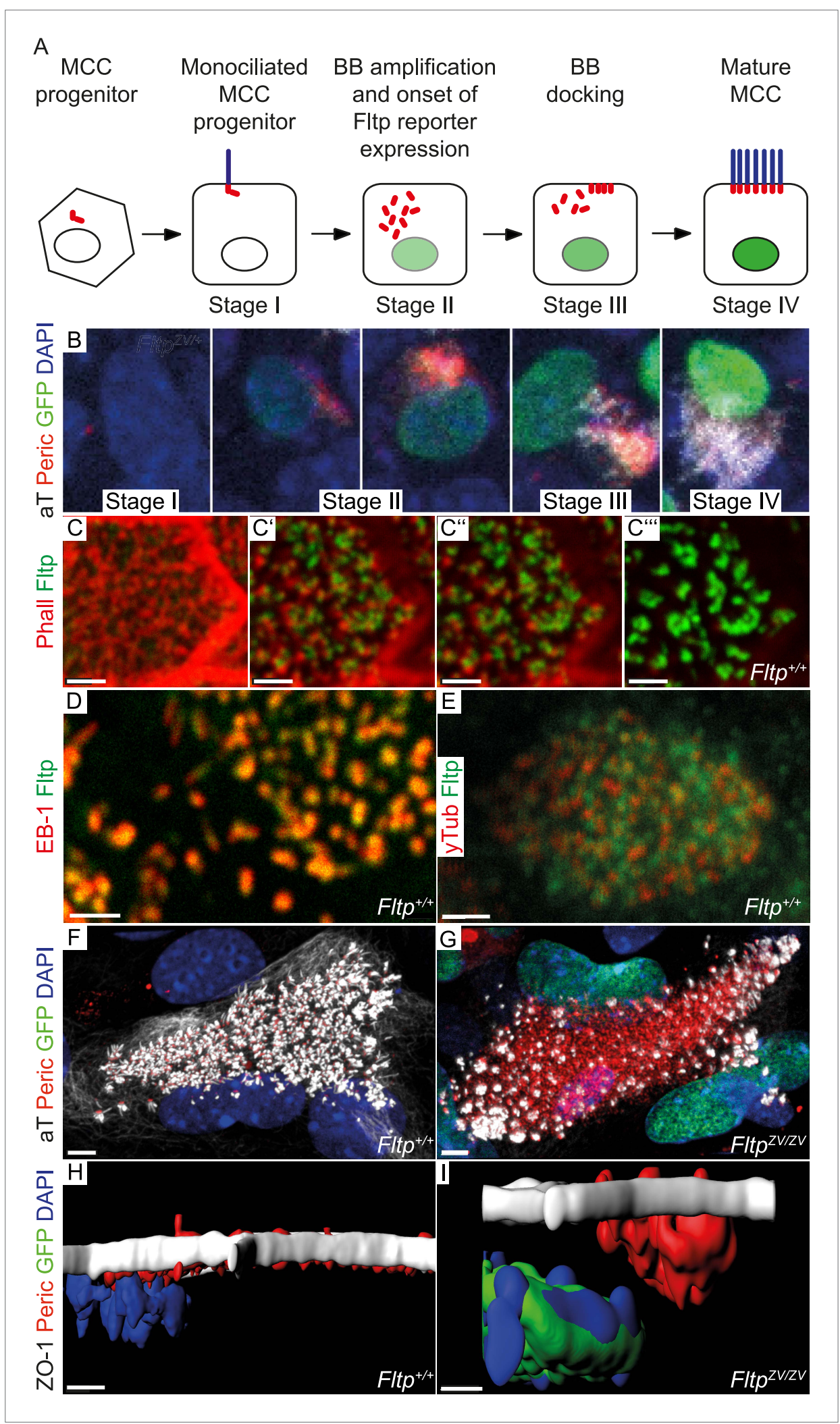

Figure 4. Fltp is expressed and necessary during BB docking. (A) Scheme of multiciliated cell (MCC) maturation. A MCC progenitor projects a primary cilium at the apical surface (stage I). Centrosome amplification is the first sign of differentiation (stage II) followed by apical transport and docking of BBs (stage III). Fully differentiated cells Figure 4. Continued on next page 
Figure 4. Continued

project multiple motile cilia at the apical surface (stage IV). Staging according to Vladar and Stearns (2007). The green nucleus indicates Fltp reporter gene expression. (B-G) LSM of ALI culture of Fltp ${ }^{2 V}$ and WT mTECs. (B) Onset and level of Fltp reporter expression correlate with the onset of BB amplification and ciliogenesis (stage II-IV) in cultured ALI mTECs as shown in the scheme in (A). (C-C'"') Confocal sections of a single cell from the sub-apical actin level (C) over the apical actin level $\left(\mathbf{C}^{\prime}\right.$ and $\left.\mathbf{C}^{\prime \prime}\right)$ to the cilia level $\left(\mathbf{C}^{\prime \prime \prime}\right)$ (ALI day 4). Fltp co-localizes with the MT-plus end binding protein EB-1 (D) (ALI day 4) next to the pericentriolar matrix (E) (ALI day 4) and to cilia in multiciliated Fltp ${ }^{+/+}$cells $\left(\mathbf{C}^{\prime \prime \prime}\right)$. (F) Fltp ${ }^{+/+}$cell showing all BBs (red dots) projecting cilia (white stripes) at the apical surface (ALI day 4). (G) In many Fltp ${ }^{Z V / Z V}$ cells the majority of BBs are not docked at the level of tight junctions marked by ZO-1 and do not project cilia (ALI day 4). (H and I) Side view IMARIS surface rendering shows that all BBs are docked at the apical surface in Fltp ${ }^{+/+}(\mathbf{H})(\mathrm{ALI}$ day 4$)$ in contrast to Fltp ${ }^{2 V / Z V}$ cells where most BBs stay in the cytoplasm (I) (ALI day 4). BBs are marked by $\mathrm{Y}$-Tubulin (YTub) and pericentrin (Peric), cilia and the tubulin network by tyrosinated-Tubulin (Tyr Tub) and acetylated-Tubulin (aT), the actin network by Phalloidin (Phall), MT plus ends by EB-1, Fltp protein by Fltp116-1, nuclei by DAPI, and nuclei of Fltp reporter expressing cells by GFP. Scale bars;

$2 \mu \mathrm{m}\left(\mathbf{C}-\mathbf{C}^{\prime \prime \prime}, \mathbf{D}, \mathbf{E}\right), 5 \mu \mathrm{m}(\mathbf{F}$ and $\mathbf{G})$

DOI: $10.7554 /$ LLife. 03842.010

The following figure supplement is available for figure 4:

Figure supplement 1. ALI differentiation efficiency and quantification of BB docking defect. DOI: 10.7554/eLife.03842.011

amplification followed by BB transport and docking (stage II-III) and subsequent cilia formation (stage IV) (Vladar et al., 2012). In these cultures, Fltp-driven H2B-Venus reporter activity is activated at the transition from stage I to stage II when centrosomes/BBs are amplified and docked in heterozygous and homozygous Fltp ALI cultures (Figure 4B). Fltp-H2B-Venus expression increases during maturation until cells are terminally differentiated. This correlates with active PCP signaling and initial asymmetric core component localization (Vladar et al., 2012). Subcellular co-localization studies further revealed that Fltp is localized to the apical, but not sub-apical actin cytoskeleton and fills the gaps in the actin network from where MT-based cilia project (Figure $4 C-C^{\prime \prime}, E$ ). Moreover, Fltp co-localizes with newly synthesized MT plus ends that are labeled with anti-EB1 antibodies (Figure 4D). Together with the direct adjacent localization of Fltp next to the pericentriolar matrix stained by $\mathrm{Y}$-Tubulin (Figure 4E), these data suggest that Fltp connects BBs and ciliary MT plus ends to the cortical actin cytoskeleton. To test this idea, we examined mTECs switched to ALI conditions from Fltp ${ }^{+/+}$and Fltp ${ }^{Z V / Z V}$ mice at day two and four of differentiation qualitatively and quantitatively. Fltp ${ }^{+/+}$and Fltp ${ }^{Z V / Z V}$ differentiated with comparable efficiencies and speed as measured by Fltp-driven H2B-GFP expression and centrosome amplification (Figure 4-figure supplement 1A), and a comparable number of centrosomes are formed in differentiating Fltp ${ }^{+/+}$and Fltp ${ }^{Z V / Z V}$ mTECs (Figure 4F, G), whereas BB docking and cilia formation was delayed at ALI+2 and +4 days of differentiation (Figure 4F-I, Figure 4-figure supplement 1B). Taken together, these data suggest that Fltp functions in the process of BB docking and cilia formation.

\section{Fltp regulates kinocilium positioning and stereocilia bundle morphogenesis in the inner ear}

To analyze the function of Fltp in PCP, BB positioning, and cilia formation in vivo, we employed the best-characterized mammalian PCP model system, the organ of Corti in the IE. Morphologically the IE looked normal and the cochlear duct is not significantly shortened or widened in Fltp ${ }^{Z V /+}$ and Fltp ${ }^{Z V / Z V}$ mice, indicating that PCP-mediated convergent extension movements are not affected at PO (data not shown). However, significant deviations of the polarized arrangement of stereocilia bundles can be seen in $\mathrm{IHC}$ and $\mathrm{OHC}$ rows with increasing severity closer to the apex along the apico-basal axis (Figure 5B-C, J-N). In addition, scanning electron microscopy (SEM) analysis uncovered severe stereocilia bundle morphogenesis defects and mispositioning of the MT-based kinocilium, very similar to phenotypes observed in mutants of spindle positioning proteins, such as $\mathrm{Ga}_{\mathrm{i}}, \mathrm{mPins}$, mlnsc, and LGN (Figure 5D-I) (Ezan et al., 2013; Tarchini et al., 2013).

To seek first evidence if Fltp functions in the PCP pathway, we investigated a potential genetic interaction of Fltp with the core PCP component Celsr1 (Curtin et al., 2003). As Fltp and Celsr1 are co-expressed in the cochlea of the IE (Shima et al., 2002), we compared stereocilia bundle morphogenesis in Celsr1 $1^{\text {crsh/crsh }}$ single mutant and Fltp ${ }^{Z V / Z V}$; Celsr1 ${ }^{\text {crsh/crsh }}$ double mutant cochleae at E18.5. On 


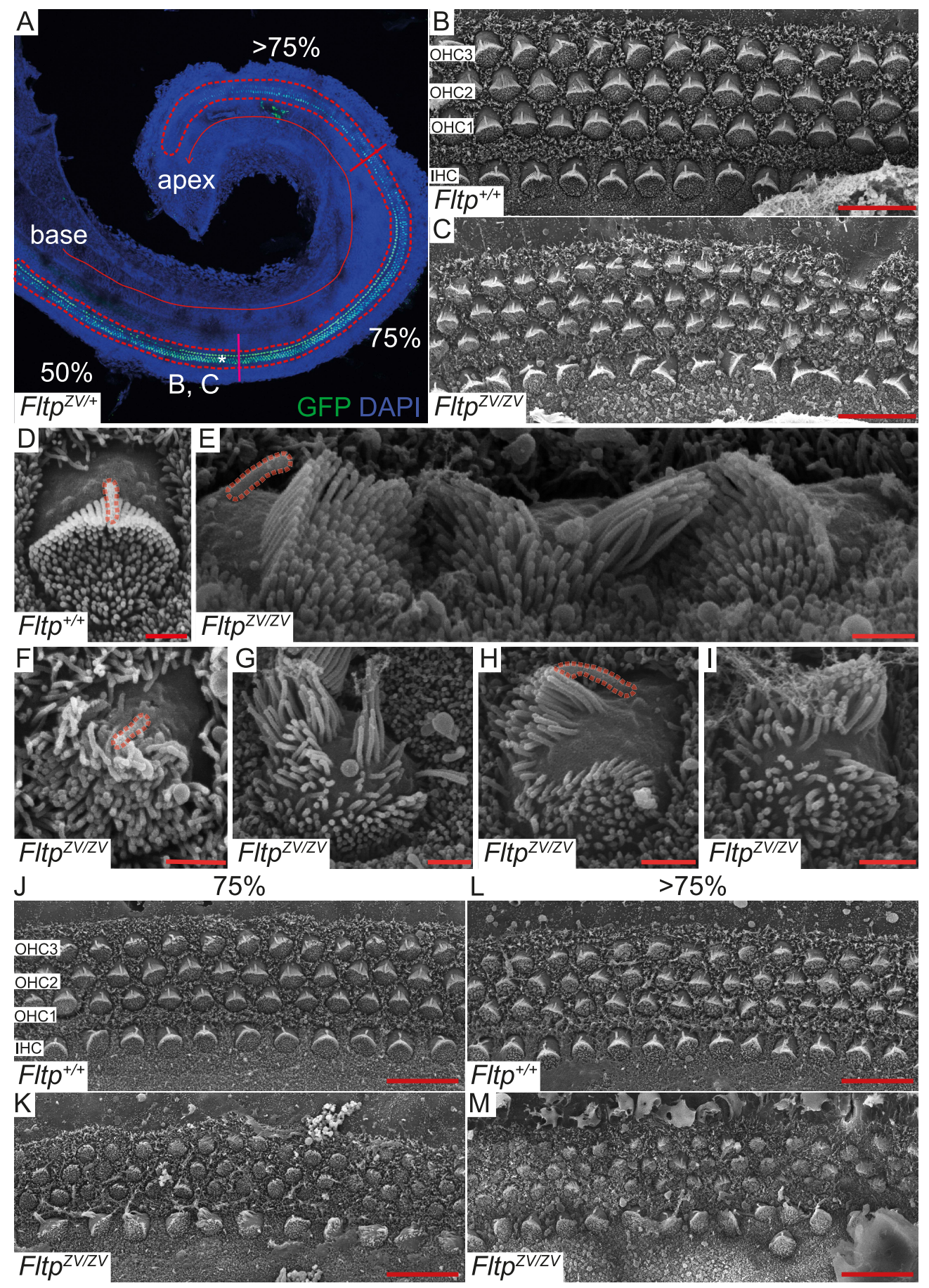

$\mathrm{N}$ Deviation of hair cells $<50 \%$
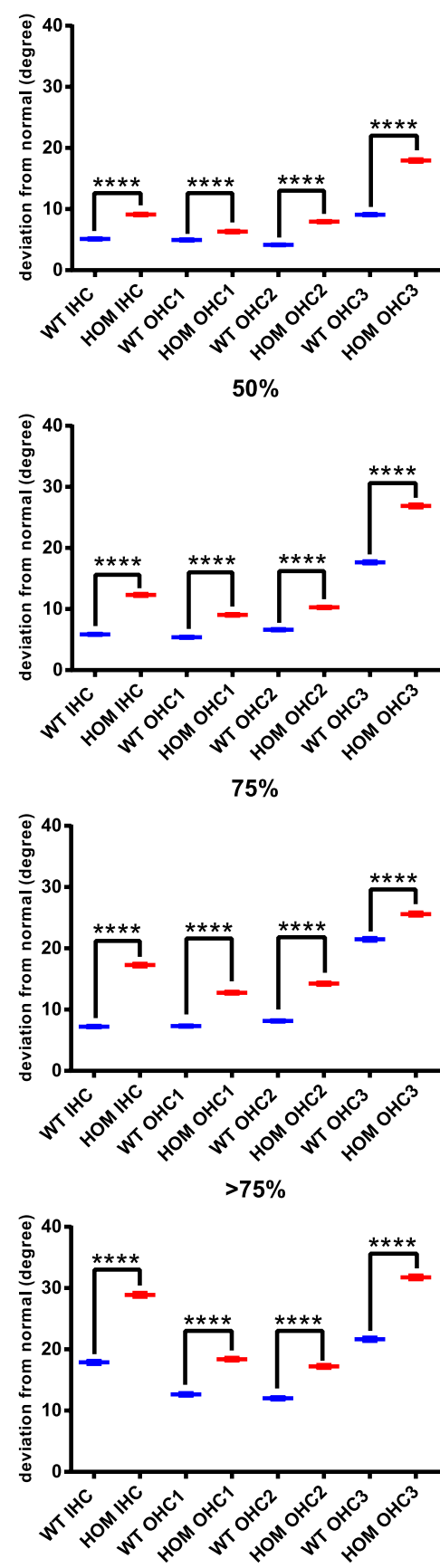

Figure 5. Fltp is required for kinocilium positioning and stereocilia bundle formation. (A) Whole-mount LSM of an Fltp ${ }^{2 V /+}$ organ of Corti (OC) showing its different regions. Fltp reporter expression is restricted to all sensory $\mathrm{HCs}(\mathrm{IHC}$ and $\mathrm{OHC}$ ) of the OC (red dashed line). (B-M) Whole-mount SEM pictures of an E18.5 OC. (B and C) Fltp ${ }^{+/+}$(WT) HCs at $50 \%$ of the OC length are perfectly polarized (B) whereas Fltp $2 V / Z V$ (HOM) HCs show orientational defects (C). (D-I) Enlargements of HCs reveal perfect polarized kinocilia as well as highly ordered stereocilia in WT (D). HOM cells are misaligned and show split stereocilia bundles $(\mathbf{E}, \mathbf{G}-\mathbf{I})$, general bundle morphology defects $(\mathbf{E}-\mathbf{I})$, as well as detached kinocilia (E). Polarity and stereocilia bundles disruption increases in HOM hair cells from 75\% (K) to $>75 \%$ (M) of OC length compared to WT cells ( $\mathbf{J}$ and $\mathbf{L}$ ). (N) Deviation of HCs from the polarity axis for WT and $\mathrm{HOM}$ animals significantly differs in all $\mathrm{OC}$ regions $(<50 \%, 50 \%, 75 \%,>75 \%)$. Quantification of $\mathrm{HC}$ rotation was performed by measuring the angle from the normal tissue polarity (measured by the medial to distal alignment of the $\mathrm{HC}$ rows) to the middle of the stereocilia bundle. In total we examined Figure 5. Continued on next page 
Figure 5. Continued

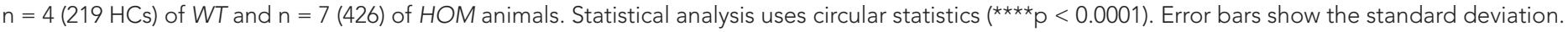
Kinocilia are marked by the red dashed lines, nuclei by DAPI, and nuclei of Fltp reporter expressing cells by GFP. Abbreviations: inner hair cell (IHC), outer hair cell (OHC1-3). Scale bars; $10 \mu \mathrm{m}$ (B and C), 1 mm (D-I).

the mixed genetic background analyzed, Celsr $1^{\text {crsh/crsh }}$ animals showed rather normal bundle alignment in $\mathrm{IHCs}$ and $\mathrm{OHCs}$ from the base to the middle part of the cochlea $(<50 \%)$, but the alignment was significantly disturbed closer to the apex (>50\%) (Figure $6 A, C, D)$. In contrast, Fltp ${ }^{\text {ZV/ZV. }}$; Celsr1 ${ }^{\text {crsh/crsh }}$ double mutant cochleae revealed an increased degree of misaligned stereocilia bundles, most pronounced in the $<50 \%$ region, and showed an additional fourth $\mathrm{OHC}$ row (Figure $6 \mathrm{~B}-\mathrm{D}$ ). These data suggest that Fltp and the core PCP gene Celsr1 either act together in the PCP pathway or in two parallel pathways important for the morphogenesis of the cochlea. To clarify if Fltp is necessary to establish PCP in the IE according to the current model (Figure 6E) (Ezan and Montcouquiol, 2013), we further investigated the localization of core PCP components. No differences in the asymmetric distribution of Vangl1 and Dvl2 at the cell cortex along the medio-lateral axis were detected in Fltp ${ }^{+/+}$and Fltp ${ }^{Z V / Z V}$ cochleae at E18.5 (Figure 6F-I), consistent with a function of Fltp downstream of core PCP molecules.

\section{Dlg3 and Fltp regulate BB positioning in the inner ear}

BB positioning and stereocilia bundle morphogenesis depend on spindle positioning proteins (Ezan et al., 2013; Tarchini et al., 2013), but how these connect to core PCP molecules and actin-rich stereocilia bundles is unknown. Due to PCP defects in the IE (Van Campenhout et al., 2011), we hypothesized that the mammalian scaffold protein Dlg3 might be a cortical adaptor for PCP-mediated BB positioning. Due to the lack of good antibodies and tools, we generated a transgenic mouse line that constitutively expresses a Venus fluorescent protein fused to the N-terminus of Dlg3 (Dlg3-Venus) (see Supplemental 'Materials and methods'). Next, we examined the subcellular localization of the Dlg3-Venus fusion protein in relation to BB, kinocilium, stereocilia bundle, core PCP molecules, and TJs in cochlea HCs using LSM and three-dimensional reconstruction (Figure 7A-B', $F-H$ ). Dlg3 specifically localizes in a crescent shape to the bare zone around the $\mathrm{BB}$ and co-localizes with the core PCP component Dvl2 at lateral TJs (Figure $7 \mathbf{A}-\mathbf{B}^{\prime}, \mathbf{G}$ ), the area where $\mathrm{mPins} / \mathrm{LGN}, \mathrm{mlnsc}$, and $\mathrm{Ga}_{i}$ are localized (Ezan et al., 2013; Tarchini et al., 2013). This suggests that Dlg3 might have a conserved function in spindle/BB positioning (Johnston et al., 2009). Interestingly, Fltp localizes at the interface between Dlg3 and the actin-based stereocilia bundles (Figure $7 C-E^{\prime}$ ), further suggesting that Fltp might be an adaptor protein linking spindle/BB positioning proteins to the apical actin cortex.

We tested this idea by analyzing Dlg3 and BB localization in Fltp mutants. When compared to WT (Figure 8A-D',J, Figure 8-figure supplement 1A), the lateral crescent of Dlg3 in Fltp mutants is disturbed and BBs are not correctly positioned in the middle of the apical Dlg3 crescent (Figure $8 E-H^{\prime}, J$, Figure 8-figure supplement 1A), implicating that Fltp binds to Dlg3 to position BBs, kinocilia, and stereocilia bundles. If Dlg3 and Fltp cooperate to position BBs, one would predict that these proteins physically interact. Dlg3 contains a central SH3 domain which could bind to the SH3 binding or PRR domain of Fltp. We tested this by co-immunoprecipitation experiments of Streptavidin-Flag (SF)-tagged Dlg3 variants and Fltp-myc variants from serum starved and ciliated HEK293T cells (Figure 9A,B). These experiments suggest a physical interaction of Fltp with Dlg3, which requires the highly conserved $\mathrm{N}$-terminal SH3 binding domain of Fltp, but not the SH3 domain of Dlg3 (Figure 9B). As Dvl2 localizes directly adjacent to Dlg3 and Fltp in the inner ear, we next examined the association of endogenous Dvl2 and $\mathrm{y}$-Tubulin with overexpressed SF full-length Fltp and SF-Dlg3 (Figure 9C). The interaction of Dvl2 with Dlg3 and Fltp and $\mathrm{y}$-Tubulin with Dvl2 and Dlg3 suggests that these proteins form a complex during $\mathrm{BB}$ transport and positioning. Finally, we tested the predicted requirement of Dlg3 for BB positioning by knock-out analysis (Figure $8 K, \mathrm{~L}$, Figure 8-figure supplement 1B,C). Taken together, these results suggest that Dlg3 is a BB positioning protein that is anchored at apical TJs (Van Campenhout et al., 2011) and cooperates with Fltp to position BB at the apical cortex of HCs in the cochlea at E18.5.

\section{Discussion}

Activation of the PCP pathway results in rearrangement of the actin and MT cytoskeleton via tissue specific effector molecules. Although actin and MT interactions need to be thoroughly controlled in 
A

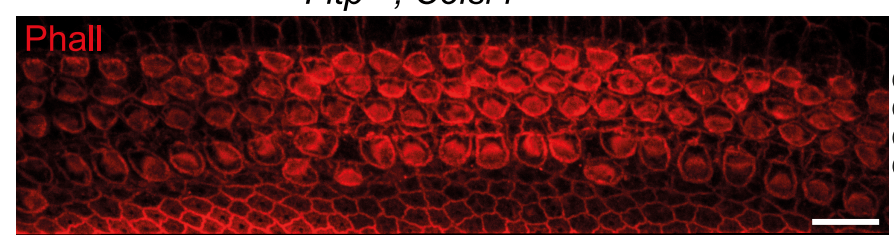

B

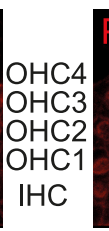

Fltp ${ }^{z V / Z v} ;$ Celsr1 ${ }^{\text {crsh/crsh }}$

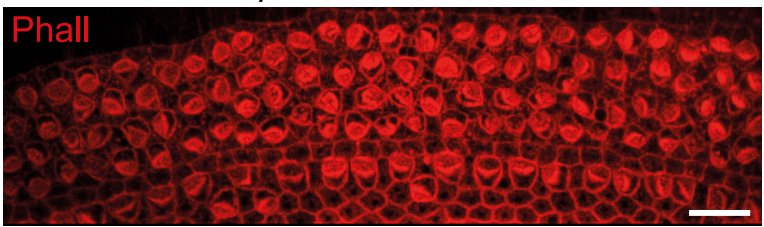

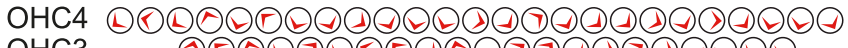

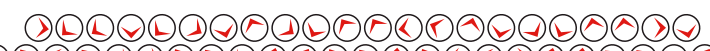

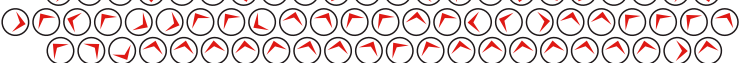

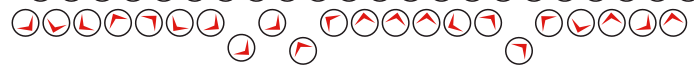

$\mathrm{OHC} 3$

$\mathrm{OHC} 2$

$\mathrm{OHC} 1$

$\mathrm{IHC}$

D

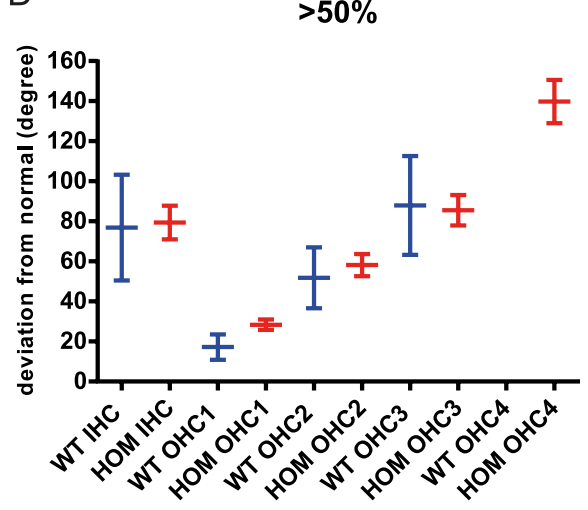

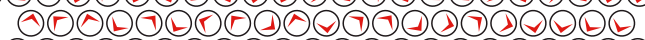

00000000000000000000000

(1) 000000000000000000000

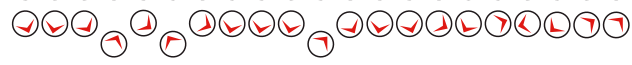

C

$<50 \%$
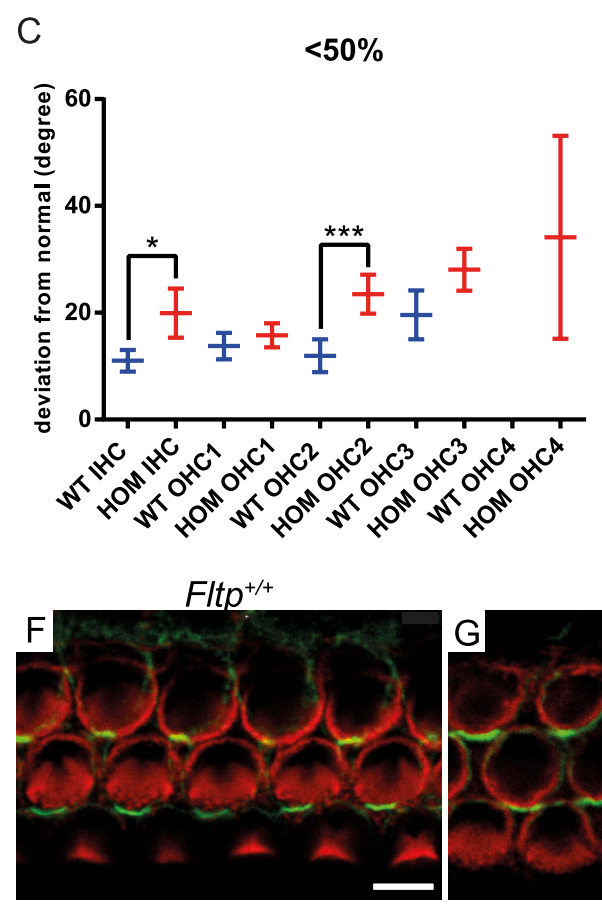

Phall Vangl1

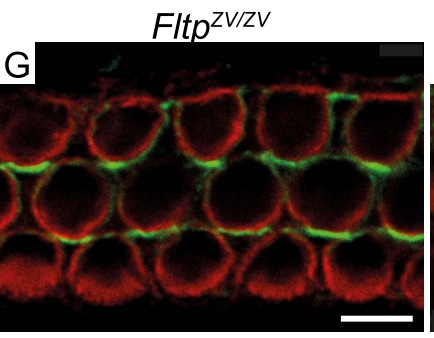

$F l t p^{Z V / Z V}$

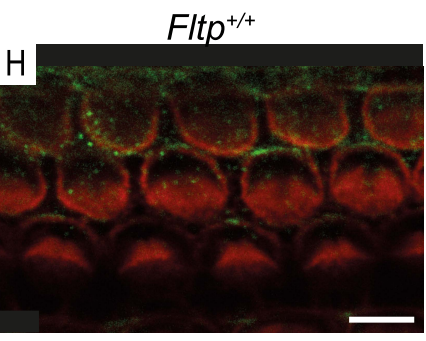

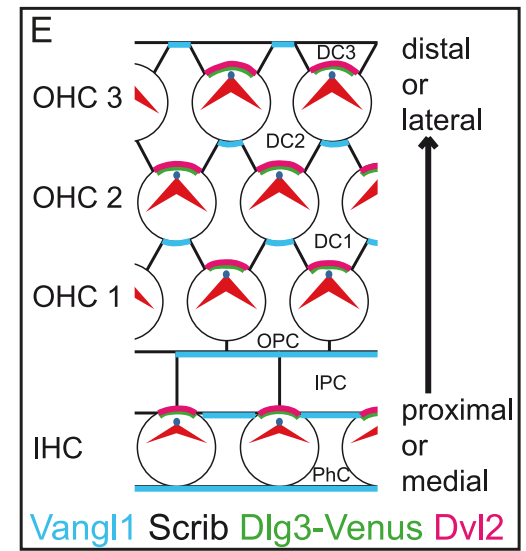

Phall Dvl2

Figure 6. Fltp is a potential downstream mediator of PCP signaling. (A-B) LSM of an E18.5 Fltp ${ }^{+/}$; Celsr1 ${ }^{\text {crsh/crsh }}$ (WT) organ of Corti (OC) (A) revealed

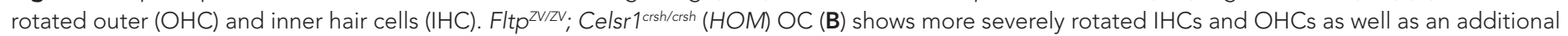
$\mathrm{OHC}$ row in comparison to (A). (C and D) Hair cells of HOM (red) animals show a more pronounced PCP phenotype compared to WT (blue) in the region $<50 \%$ and $>50 \%$ of the OC (for cochlea region nomenclature and quantification method see Figure 5). In total we analyzed $n=1(130$ cells) at $<50 \%$, $n=1$ (90) at $>50 \%$ for WT, $n=5(298)$ at $<50 \%, n=5$ (743) at $>50 \%$ for HOM. Statistical analysis uses a Kruskal-Wallis test ( $\left.{ }^{*} p=0.0375 ;{ }^{* \star \star} p=0.0003\right)$. Error bars show the $95 \%$ confidence interval of the mean. (E) Model illustrating PCP molecule localization in IE hair cells. (F-I) Whole-mount IE (E18.5) LSM of $3 \mathrm{OHC}$ rows revealed Vangl1 localization at the lateral side of supporting cells in Fltp ${ }^{+/+}(\mathbf{F})$ and Fltp ${ }^{2 V / Z V}(\mathbf{G})$ animals and Dvl2 localization at the lateral side of IE hair cells in Fltp ${ }^{+/+}(\mathbf{H})$ and Fltp ${ }^{Z V / Z V}(\mathbf{I})$ animals indicating that core PCP protein localization is not disrupted. The actin network and stereocilia are marked by Phalloidin (Phall) and core PCP proteins by Vangl1 and Dvl2. Abbreviations: inner phalangeal cells (PhC), inner pillar cells (IPC), outer pillar cells (OPC), Deiters' cells (DC1-3). Scale bars; $10 \mu \mathrm{m}$ (A and $\mathbf{B}), 5 \mu \mathrm{m}(\mathbf{F}-\mathbf{I})$.

DOI: 10.7554/eLife.03842.013

virtually all cell types in different cell cycle phases across species from yeast to humans, it is surprising that not many adaptor proteins that mediate such interactions are identified (Rodriguez et al., 2003). These global cytoskeletal rearrangements underlie many fundamental morphogenetic processes in which cellular asymmetries need to be established and maintained, such as tissue morphogenesis, cell migration, or BB and spindle positioning in post-mitotic and mitotic cells, respectively (Wallingford, 2012). We describe the identification and functional analysis of Fltp, a protein that orchestrates cytoskeletal rearrangements in cells that acquire PCP. Our data suggest, that 


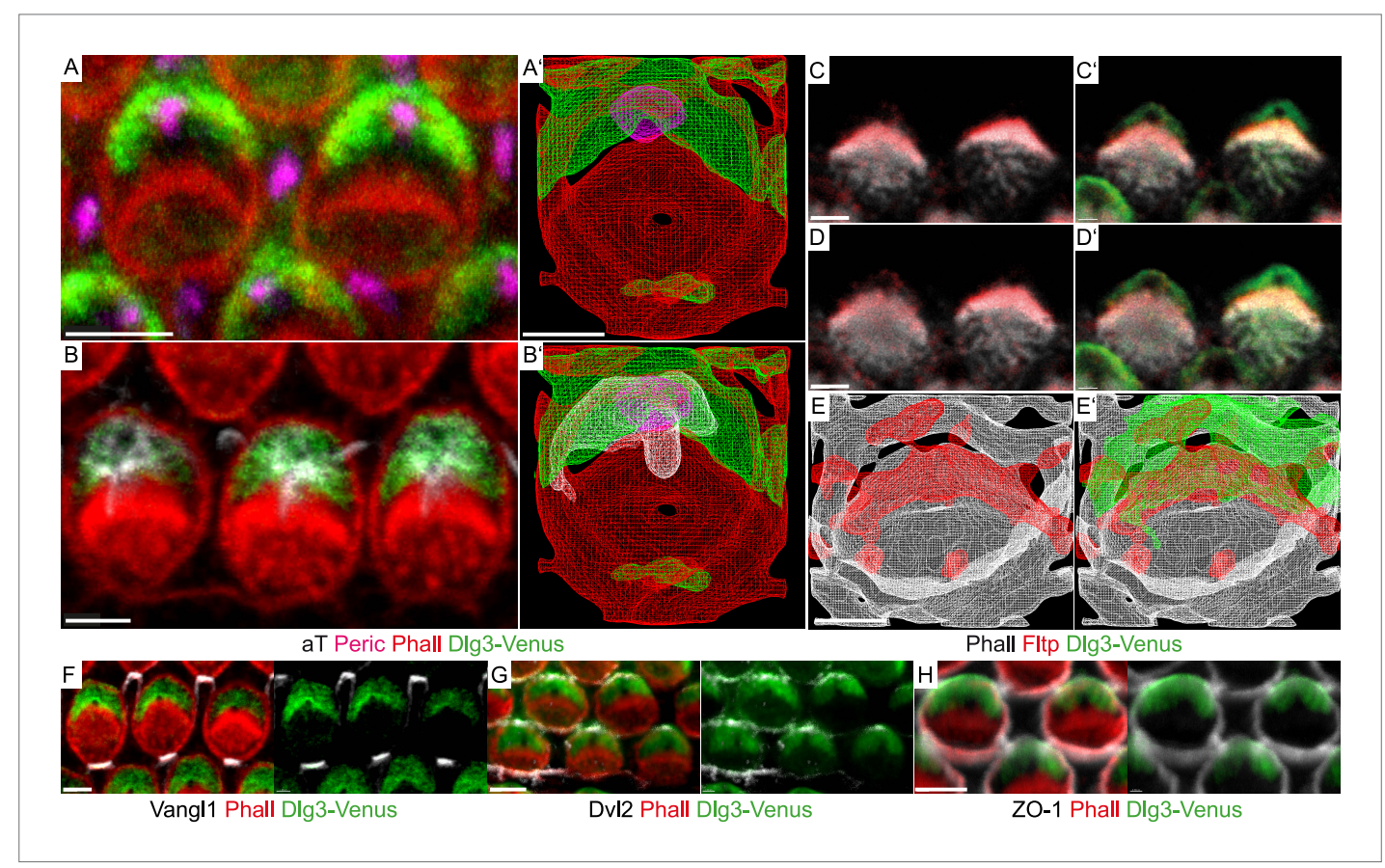

Figure 7. Fltp is located at the interface of apical actin and Dlg3 in IE hair cells. (A, B, C-D', F-H) Single section LSM of outer HCs of an Fltp ${ }^{+/+}$; Dlg3-Venus animal at E18.5 reveals that Dlg3-Venus is located at the lateral membrane and at the medial membrane (or the lateral membrane of the supporting cell) of IE HCs (A and $\mathbf{B}$ ). Fltp is localized lateral to the cuticular plate (CP) and the stereocilia bundles (SC) (C and D). Dlg3-Venus is located in a lateral crescent overlapping with Fltp localization $\left(\mathbf{C}^{\prime}\right.$ and $\left.\mathbf{D}^{\prime}\right)$. The unstained area marks the region of the BB $\left(\mathbf{C}^{\prime}\right.$ and $\left.\mathbf{D}^{\prime}\right)$. Dlg3-Venus is co-localized with $\mathrm{Dvl} 2(\mathbf{G})$ at the most lateral membrane directly opposite of Vangl1 (F) and with ZO-1 at the apical membrane (H). ( $\left.\mathbf{A}^{\prime}, \mathbf{B}^{\prime}, \mathbf{E}, \mathbf{E}^{\prime}\right)$ IMARIS wireframe animation of a Dlg3-Venus IE HC showing Dlg3-Venus co-localization with the BB, the kinocilium, and actin $\left(\mathbf{A}^{\prime}\right.$ and $\left.\mathbf{B}^{\prime}\right)$ and a Fltp ${ }^{+/+}$; Dlg3-Venus IE HC showing Fltp, Dlg3-Venus, and Phalloidin co-localization (E and $\left.\mathbf{E}^{\prime}\right)$. The actin network, the CP, and the SC are marked by Phalloidin (Phall), Fltp protein by Fltp116-1 (Fltp), the kinocilium by acetylated-Tubulin (aT), the BB by pericentrin (Peric), the apical cell membrane by ZO-1, core PCP proteins by Vangl1 and Dvl2, and Dlg3-Venus fusion protein by GFP. Scale bars; $2 \mu \mathrm{m}\left(\mathbf{A}-\mathbf{E}^{\prime}\right), 3 \mu \mathrm{m}(\mathbf{F}-\mathbf{H})$.

DOI: 10.7554/eLife.03842.014

Fltp is important for BB docking and positioning in mammals through regulating actin and MT interactions.

\section{Fltp is a potential PCP effector gene that regulates MT-actin interactions in mammals}

Several lines of evidence suggest that Fltp is a potential PCP effector regulating the cytoskeleton. First, the Fltp gene and reporter are expressed in several tissues known to depend on active PCP signalling, such as the node, ependymal cells, floor plate, lung, eye, and IE. Second, Fltp expression kinetic correlates with dynamic PCP acquisition, terminal differentiation, and cellular morphogenesis in the lung and the IE. Third, the double knock-out of Fltp and the core PCP gene Celsr1 show a more severe PCP phenotype than both single mutants, raising the possibility that both genes act in the same pathway, which needs further confirmation. If this is the case, then Fltp seems to be a downstream effector molecule in the PCP pathway, as it is not required for PCP establishment, but rather for cell intrinsic PCP transduction leading to cytoskeletal rearrangements. This is supported by the fact that phenotypes observed for the PCP effectors Intu and Fuz in the mucociliary epithelium of the frog and Fltp in the murine lung are strikingly similar (Park et al., 2006, 2008). Additionally, spindle positioning rather than core PCP mutant phenotypes mirrors the Fltp mutant phenotype in the IE (Ezan et al., 2013; Tarchini et al., 2013). Finally, Fltp is localized at the interface of spindle positioning complexes that capture MT plus ends and connect to the PM and the apical actin cytoskeleton. Loss of Fltp leads to failure in BB docking and positioning most probably through disconnection 

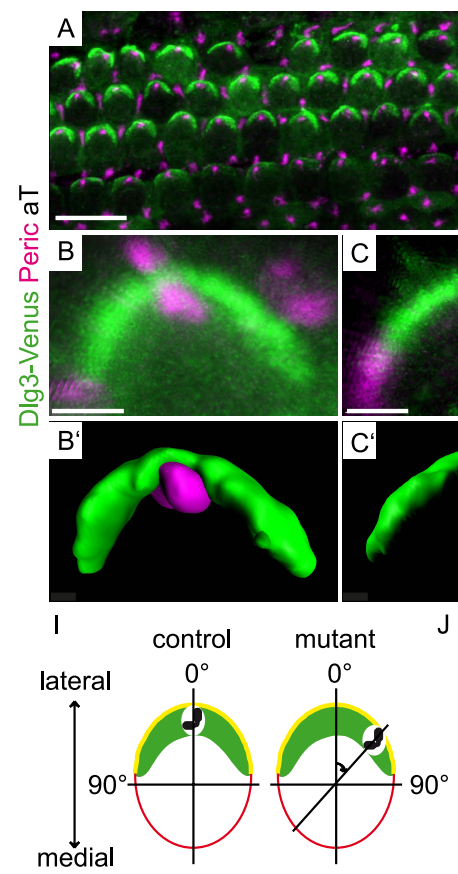

apical membrane of hair cell Dlg3-Venus

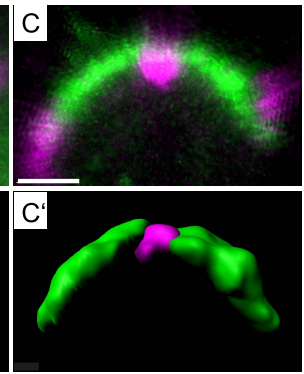

$\mathrm{J}$ Basal body position in the Dlg3 crescent $\mathrm{K}$

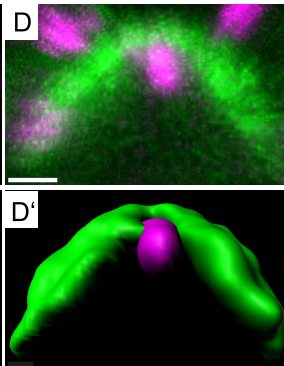

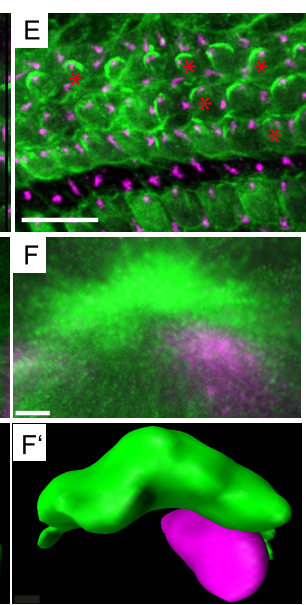

$D / g 3^{-/}$

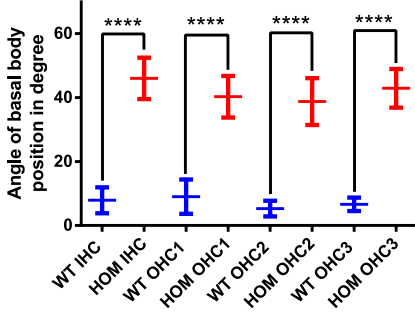

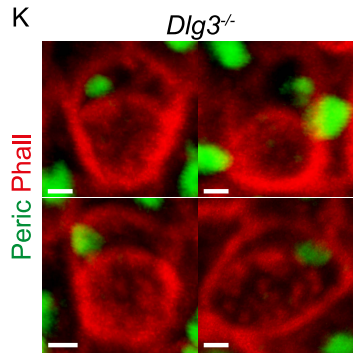
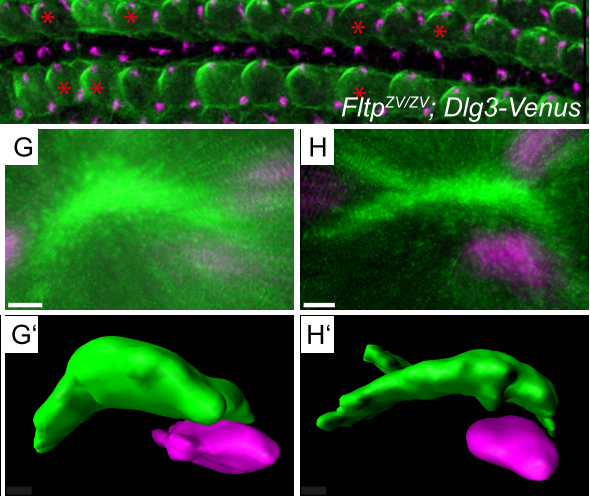

$\mathrm{L}$ Basal body position relative to stereocilia

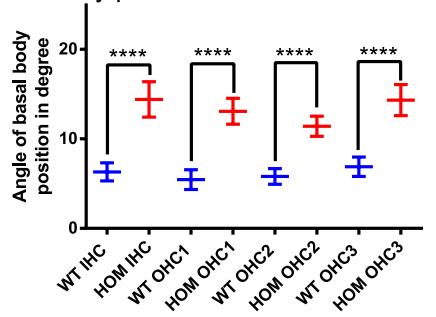

Figure 8. Loss of Fltp or Dlg3 leads to BB mispositioning in IE hair cells. (A-D) LSM of Fltp+/+; Dlg3-Venus (WT) IE HCs at E18.5 reveals BBs in the middle of the lateral Dlg3-Venus crescent. (B'-D') IMARIS surface rendering pictures of WT HCs. (E-H) LSM of Fltp2VIZv; Dlg3-Venus (HOM) IE HCs at E18.5. BBs are located at the edge of the lateral Dlg3-Venus crescent. The crescent itself often shows defective localization. The red asterisk marks some affected cells. $\left(\mathbf{F}^{\prime}-\mathbf{H}^{\prime}\right)$ IMARIS surface rendering pictures of HOM HCs. For quantification see Figure 8-figure supplement 1A. (I) For BB mispositioning analyses the angle between the middle of the Dlg3-Venus crescent and the BB location was measured. (J) BB position in affected HCs of HOM animals significantly differs from the position in the WT. In total we analyzed $\mathrm{n}=2$ (73 cells) for WT and $\mathrm{n}=2$ (119) for HOM. (K) LSM of Dlg $3^{-/-}$(Dlg $3^{\text {tm } 1 \mathrm{Grm} \text { ) }}$ IE HCs reveals mislocalized BBs and rotated hair cells. For quantification Figure 8-figure supplement 1B,C. (L) Analysis of BB mispositioning was performed as described in (I). BB position in affected $\mathrm{HCs}$ of $\mathrm{Dlg} 3^{-/-}(\mathrm{HOM})$ animals significantly differs from the Dlg $3^{+/+}(\mathrm{WT})$ position. In total we analyzed $n=7$ (470 cells) for WT and $n=13$ (804) for HOM. Statistical analysis uses an one-way ANOVA (J) or a Kruskal-Wallis test (L) (**** $<0.0001)$. Error bars show the 95\% confidence interval of the mean. The actin network and stereocilia are marked by Phalloidin (Phall), BBs by pericentrin (Peric), cilia by acetylated-Tubulin (aT), and Dlg3-Venus fusion protein by GFP. Abbreviation: inner hair cell (IHC), outer hair cell (OHC 1-3). Scale bars; 10 $\mu$ m (A and $\mathbf{E}), 1 \mu \mathrm{m}\left(\mathbf{B}-\mathbf{D}^{\prime}, \mathbf{F}-\mathbf{H}^{\prime}, \mathbf{K}\right)$

DOI: 10.7554/eLife.03842.015

The following figure supplement is available for figure 8:

Figure supplement 1. BB mispositioning in Fltp ${ }^{2 v}$; Dlg3-Venus and Dlg3 animals.

DOI: 10.7554/eLife.03842.016

of the BB, MT, and actin cytoskeleton in the mammalian lung and IE. Although the mechanisms of Fltp action in these two tissues might differ, a common theme seems that Fltp acts at the interface of the MT and actin cytoskeleton for BB docking and positioning, which we discuss in the following sections.

\section{Fltp regulates $B B$ docking and cilia formation in the lung}

PCP is best studied in the mammalian IE, but is also apparent in MCCs of the lung and mucociliary epithelium of the frog skin (Wallingford, 2012). In both terminally differentiated cell types, core PCP molecules are asymmetrically localized the apical PM and translate long-range PCP signals into cell intrinsic cytoskeletal changes that coordinate BB docking and positioning as well as cilia formation in mono- and multiciliated cells. In MCCs of the frog skin the PCP effector proteins Intu and Fuz have been shown to control ciliogenesis and together with Dvl control apical docking and planar polarization of BBs by coordinating apical actin assembly (Park et al., 2006, 2008). It was further suggested that the PCP effector Fuz and Dvl2 control membrane trafficking and vesicle fusion at the BB essential 


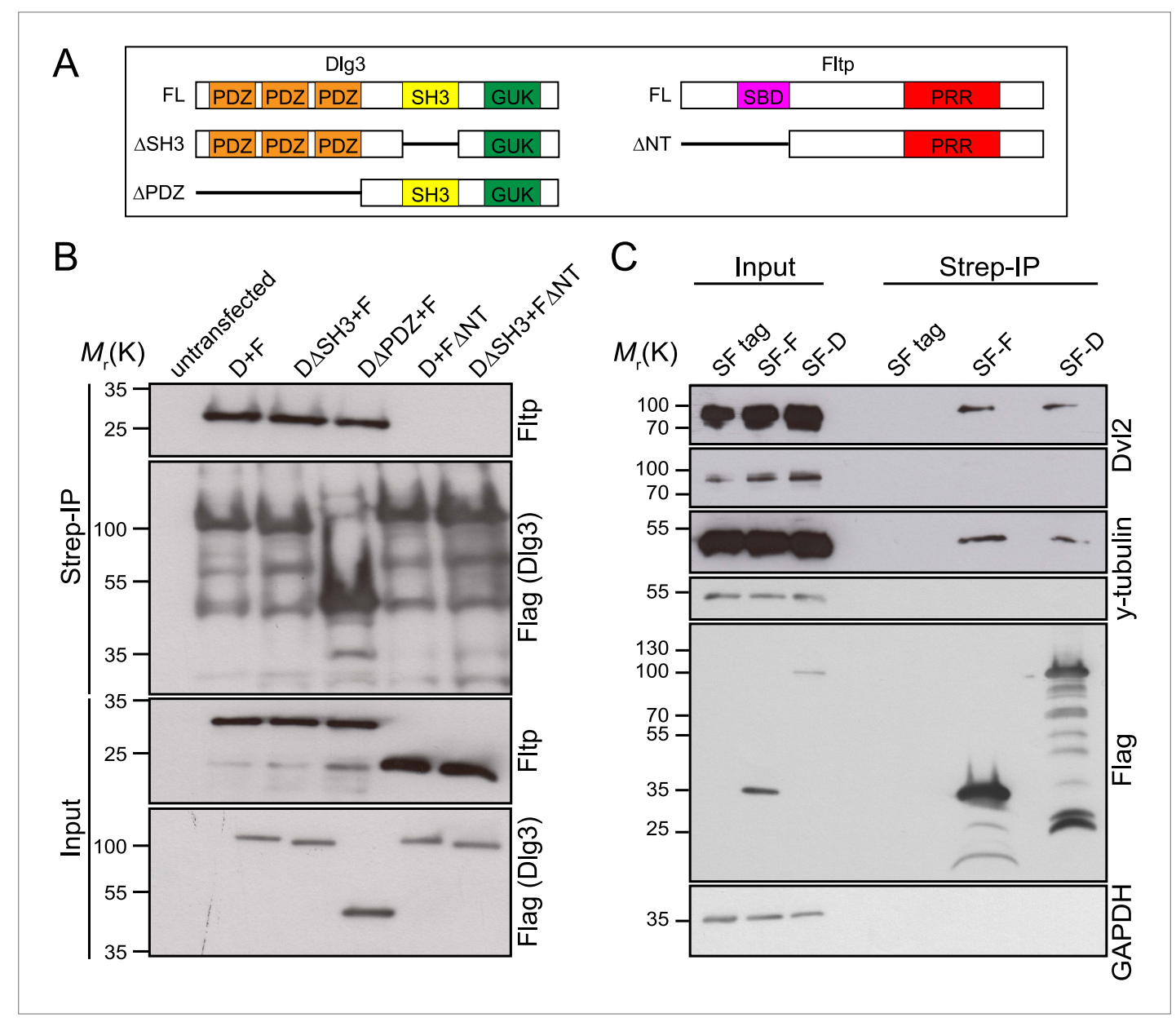

Figure 9. Fltp interacts with proteins associated with the TJ complex (Dlg3), the BB ( $y$-Tubulin) and with the core PCP protein Dvl2. (A) Dlg3 and Fltp constructs used for interaction domain mapping. (B) N-terminus of Fltp is essential for interaction with Dlg3. HEK293T cells were transfected with SF Dlg3 variants and with Fltp-myc variants. SF-tagged Dlg3 was immunoprecipitated using Streptavidin beads (Strep-IP). Full-length Fltp-myc was detected in the Strep-IP in the presence of full-length SF-Dlg3, SF-Dlg3 $\Delta$ SH3, and SF-Dlg3 $\Delta$ PDZ. F $\Delta N T$ cannot be co-immunoprecipitated with SF-Dlg3. (C) Fltp and Dlg3 are found in a complex together with Dvl2 and Y-Tubulin. HEK293T cells were transfected with SF Dlg3 and SF Fltp. In a Strep-IP for Fltp and Dlg3, endogenous Dvl2 and $\mathrm{Y}$-Tubulin were co-immunoprecipitated. Abbreviations: D: Dlg3; F: Fltp; SBD: SH3 binding domain; PRR: proline rich repeat. DOI: 10.7554/eLife.03842.017

for ciliogenesis (Park et al., 2008; Gray et al., 2009). Studying Fltp function in the ALI primary cell culture system allowed us to link the onset of Fltp expression to the time of BB amplification, docking, and cilia formation. In MCCs, we have shown a close association of Fltp with the apical actin-MT cytoskeleton and BBs. Fltp localization at the apical surface is reminiscent of Dvl, Sec8, and Intu localization in frog multiciliated mucociliary epithelium (Park et al., 2006). Lack of Fltp function leads to BB docking defects and reduced number of cilia formed at the apical PM in ALI cultures and in vivo. Fltp physically interacts with Dlg3 and the core PCP molecule Dvl2, which regulates BB transport together with proteins of the secretory pathway (Park et al., 2008). We have previously shown that Dlg3 is the only Dlg family member that is monoubiquitinated by Nedd4 and Nedd4-like E3 ligases. This is required for apical PM transport mediated by the motor protein Dynein IC and Sec8, a component of the exocyst complex (Van Campenhout et al., 2011). Thus, taken together, these findings suggest that Fltp cooperates with Dlg and Dvl family members to regulate apical BB transport and docking of $\mathrm{BBs}$ in multiciliated tissues. However, there is likely not a strong parallel between $\mathrm{BB}$ docking in $\mathrm{MCC}$ s and $\mathrm{BB}$ positioning in the $\mathrm{IE}$, a topic which needs to be addressed in the future. 


\section{Fltp and the spindle positioning protein Dlg3 act in concert to position BBs in the IE}

To better understand the function of Fltp, we further focused our analysis on the IE as a well-described model for PCP. It was previously shown that core PCP molecules localize to distinct apical membrane compartments, but differential localization seems not sufficient to instruct morphogenesis of actin-rich hair bundles (Jones and Chen, 2008). Instead, BB as well as kinocilium formation and positioning is essential for hair bundle morphogenesis, which is regulated by opposing localization of spindle positioning proteins and apical polarity proteins (Ezan et al., 2013; Tarchini et al., 2013). mlnsc, mPins/ LGN, and $\mathrm{Ga}_{i}$ localize in a microvilli-free zone (bare zone) at the lateral side, whereas the apical polarity complex consisting of Partitioning defective 3 and $6(\mathrm{Par} 3,6)$ as well as atypical protein kinase $C$ (aPKC) localize at the medial side. Surprisingly, localization of spindle proteins was not affected in the classical PCP mutants (Ezan et alo, 2013), which either suggests redundancy in the PCP pathway or alternative mechanisms that position spindle proteins and BBs in the IE. We provide evidence that Fltp and Dlg3 position BBs and stereocilia bundles in the IE in addition to $\mathrm{Ga}_{\mathrm{i}}$, $\mathrm{mPins} / \mathrm{LGN}$, and mlnsc, suggesting that we have identified important new molecules for $\mathrm{BB} /$ spindle positioning in mammals. Dlg3 and Fltp together surround the BB and anchor this organelle asymmetrically to the apical cortex likely via Dvl2 and TJ-associated proteins on the one side and attachment to the apical actin cytoskeleton on the other side. We have recently shown that the scaffold protein Dlg3 is important for apical polarity and TJ formation by interaction with a number of TJ-associated proteins (Van Campenhout et al., 2011). In the fruit fly, Dlg, Mushroom body defective (Mud), and Pins are absolutely necessary for spindle positioning in various cell types in vitro and in vivo (Bellaiche et al., 2001; Johnston et al., 2009; Bergstralh et al., 2013). The co-localization of $\mathrm{Ga}_{\mathrm{i}}$, mPins/LGN and mlnsc, and Dlg3-Venus in the bare zone further suggests that also in mammals these proteins act in concert to position BBs and spindles. Furthermore, the basolateral polarity complex consisting of Dlg, Lgl, and Scrib is known to establish membrane domains by reciprocal inhibitory interactions with the apical aPKC-Par3/6 polarity complex and to regulate A-B spindle positioning (Knoblich, 2008; Siller and Doe, 2008). These reciprocal inhibitory interactions of the apical and basolateral polarity complex at the apical surface further stabilized the positioning of BBs at the apical PM. But how spindle positioning complexes are linked to the actin cytoskeleton is still a mystery in mammals. In yeast, MT plus ends are captured by Kar9a, which binds directly to a type $\mathrm{V}$ myosin motor bound to actin filaments (Korinek et al., 2000; Lee et al., 2000). As described above, Fltp functions very likely as a molecular adaptor protein localized between the interface of spindle positioning complexes and the apical actin cytoskeleton. Loss of Fltp function leads to disconnection of MT-based kinocilium and actin-based stereocilia bundles. Together, this suggests that Fltp couples BB/spindle positioning proteins to core PCP molecules and to the actin cytoskeleton in the IE. Further studies have to be conducted to precisely clarify how this complex is anchored to the cytoskeleton and core PCP components.

\section{Fltp function in development and disease}

Knock-out analysis of Fltp revealed an important function in the terminal differentiation of multiciliated lung cells and the morphogenesis of stereocilia bundles on IE HCs. This emphasizes that PCP acquisition and remodeling of the actin- and MT-based cytoskeleton is crucially important for the maturation and function of terminally differentiated cell-types in tissues and organs. Consequently, mutations of both core PCP and effector molecules lead to a wide spectrum of ciliary dysfunction syndromes (Gerdes et al., 2009; Wallingford, 2012). For instance, in primary cilia dyskinesia, lack of directed mucociliary clearance and recurrent respiratory tract infections can eventually progress to permanent lung damage (Storm van's Gravesande and Omran, 2005). The Fltp knock-out mouse shows BB docking and cilia formation defects in MCCs of the lung as well as terminal airway constrictions. This suggests that human FLTP might be mutated in lung disease, a hypothesis that we are currently testing with our clinical partners. Moreover, mutation of Fltp leads to a stereocilia morphogenesis defect in the cochlea of the IE, which suggests that mutation of FLTP can cause hearing loss in human. Mutations in human DLG3 are associated with X-linked mental retardation (Lickert and Van Campenhout, 2012) and we have previously identified that the scaffolding protein Dlg3 regulates apical polarity and TJ formation as well as PCP in the IE (Van Campenhout et al., 2011). In this study, we have shown that Fltp and Dlg3 cooperate in BB positioning in the IE. As Drosophila Dlg acts as a tumor suppressor regulating proliferation and asymmetric cell division (Johnston et al., 2009), it is well possible that the Fltp-Dlg3 complex is involved in similar cellular processes in mammals. Indeed, 
we have evidence that Fltp regulates cell division of intestinal stem cells (Böttcher and Lickert, in preparation), suggesting that we identified a novel molecule with brought implication for ciliary disease and stem cell-mediated tissue homeostasis.

\section{Materials and methods}

\section{Animal data}

Mouse keeping was done at the central facilities at HMGU in accordance with the German animal welfare legislation and acknowledged guidelines of the Society of Laboratory Animals (GV-SOLAS) and of the Federation of Laboratory Animal Science Associations (FELASA). Post-mortem examination of organs was not subject to regulatory authorization.

\section{Generation of Fltp antibodies}

Fltp antibodies were generated as described previously (Lange et al., 2012). Two affinity purified polyclonal antibodies (Fltp1, Fltp116-1) against mouse Fltp using the peptide sequence: DNPD EPQSSHPSAGHT for Fltp1 and KPFDPDSQTKQKKSVTKTVO for Fltp116-1 were raised in rabbit (Pineda, Berlin, Germany). The Fltp1 epitope locates to the less well conserved C-terminal PRR (Figure 1C, red empty box). The Fltp116-1 epitope (Figure 1C, red filled box) resides N-terminal to the Fltp1 epitope and is less conserved in human.

\section{Antibodies}

Primary antibodies used were rabbit anti-Fltp1 (Pineda, Berlin), rabbit anti-Fltp116-1 (Pineda, Berlin), mouse anti-ZO-1 (33-9100: Invitrogen, Carlsbad, CA), mouse anti- $\alpha-T u b u l i n$ (T6199; Sigma), mouse anti-acetylated Tubulin (T7451; Sigma), mouse anti-y-Tubulin (ab11316; Abcam), rabbit anti- $\beta$-Catenin (C2206; Sigma), chicken anti-GFP (GFP-1020; Aves Labs), rat anti-tyrosinated Tubulin (MAB1864; Millipore), rabbit anti-pericentrin (PRB-432C; Covance), rabbit anti-Vangl1 (HPA025235; Sigma), rabbit anti-Dvl2 (3216; Cell Signaling), and Alexa Fluor 546 Phalloidin (A22283; Invitrogen). Immunostainings were performed as described in the Supplemental 'Materials and methods'.

\section{Western blot}

Western blot analysis was performed by standard procedures. Following antibodies were used; mouse anti-Flag (A8592; Sigma), rabbit anti-Fltp1 (Pineda, Berlin), rabbit anti-Fltp116-1 (Pineda, Berlin), rabbit anti-Dvl2 (3216; Cell Signaling), mouse anti-y-Tubulin (ab11316; Abcam), mouse anti-GAPDH (CB1001; Merck Bioscience).

\section{X-gal (5-bromo-4-chloro-3-indolyl- $\beta$-D-galactoside) staining}

$\beta$-gal staining of whole-mount embryos and organs were performed as previously described (Liao et al., 2009). Some tissues were further processed. Not BABB treated whole-mount embryos/organs were fixed, washed in PBS, and photographed. BABB treated embryos/organs were left in BABB for photographing.

\section{SEM analysis}

For SEM, inner ears were fixed in $2.5 \%$ glutaraldehyde in cacodylate buffer and then treated using standard procedures.

\section{Generation of the Fltp ${ }^{2 \mathrm{v}}$ targeting vector}

The knock-in/knock-out construct was designed as shown in Figure 3A. 5' and 3' HR for the Fltp gene were amplified by PCR $(449,450,451,452)$ using a C57BL/6J BAC clone (RP23-333P11) as template. These two PCR products were subcloned into the pL254 vector (Liao et al., 2009). The resulting vector was digested with HindIII, Spel and electroporated into electrocompetent EL350 bacteria containing the Fltp BAC clone to retrieve the WT sequence between PCR homology arms resulting in the Fltp retrieval vector. For cloning of the knock-in/knock-out cassette in pBKS- $5^{\prime}$ and $3^{\prime}$ HR for the knock-in into the ATG of exon, two of Fltp were generated by PCR $(453,454,455,456)$ using the previously mentioned $\mathrm{BAC}$ as a template and subcloned into $\mathrm{PBKS}^{-}$using the introduced restriction sites, resulting in $\mathrm{pBKS}^{-}-\mathrm{Fltp}-\mathrm{H}_{\mathrm{N}} \mathrm{Arms}$. The targeting vector was generated by ligating the loxP flanked neomycin (neo) resistance cassette (PL-452) (Liu et al., 2003) into the pBKS--H2B-Venus-intronSV40pA plasmid resulting in $\mathrm{pBKS}^{-}-\mathrm{H} 2 \mathrm{~B}-$ Venus-intron-SV40pA-loxP-bGHpA-neo-EM7-PGK-loxP (pBKS--H2B-Venus-neo). The T2A sequence from Thosea asigna virus was introduced into the Notl site 
of pBKS--H2B-Venus-neo by annealing the following oligos 2A_fwd; $2 A \_r e v$, which created a Notl compatible overhang resulting in $\mathrm{pBKS}-\mathrm{S}^{-} \mathrm{2A}-\mathrm{H} 2 \mathrm{~B}-$ Venus-neo. NLS-lacZ (nuclear localization signal- $\beta$ galactosidase fusion protein) was ligated into the $\mathrm{pBKS}^{-}-2 \mathrm{~A}-\mathrm{H} 2 \mathrm{~B}-$ Venus-neo vector resulting in $\mathrm{pBKS}^{-}$NLS-lacZ-2A-H2B-Venus-neo. To finish the minitargeting construct, we cloned pBKS--NLS-lacZ-2A-H2BVenus-neo into pBKS--Fltp-HomArms (both cut with Notl and Sall). The minitargeting construct was cut out by Sacll and Kpnl, electroporated in EL350 bacteria, and introduced into PL254 via bacterial homologes recombination resulting in the final targeting construct (PL254-Fltp-NLS-lacZ-2A-H2BVenus-intron-SV40pA-loxP-bGHpA-neo-EM7-PGK-loxP) which was confirmed by sequencing and is ready for electroporating into embryonic stem (ES) cells (after linearization by Ascl).

\section{Generation of the Fltp knock-out mouse line and Southern blot}

To generate targeted ES cells, we electroporated IDG3.2-F1 ES cells (C57BL/6J x 129S6/SvEvTac) (Hitz et al., 2007) with the Ascl-linearized FltpZV targeting vector and neomycin resistant clones were selected using $300 \mu \mathrm{g} / \mathrm{ml}$ G418 (Invitrogen). Homologous recombination at the Fltp locus was confirmed by Southern blot analysis of Dralll-digested genomic DNA using the Fltp 5'-probe (620 bp) (5' S Fltp FWD Xhol; 5' S Fltp REV Xbal). 8 out of 100 clones (in total: 22 positive clones out of 289) showed homologous recombination. Due to restriction fragment length polymorphisms (RFLP) of the $\mathrm{Bl} / 6 \mathrm{~J}$ and $129 \mathrm{~S} 6$ alleles and an isogenic Bl6 targeting vector, homologous recombination occurred preferentially on the $\mathrm{Bl} / 6 \mathrm{~J}$ allele and reduced the size of the restriction fragment from $16.443 \mathrm{bp}$ to $11.469 \mathrm{bp}$. The $129 \mathrm{~S} 6 \mathrm{WT}$ band is smaller in size than the Bl/6J WT band and is not targeted. Both ES cell clones gave birth to $\Delta$ neo animals, but for the analysis we concentrated on one clone and founded the animal colony on this. After deletion of the neo cassette mice were backcrossed to $\mathrm{C} 57 \mathrm{Bl} / 6 \mathrm{~J}$ to eliminate the $\mathrm{Cre}$ allele. Only those mice negative for the $\mathrm{Cre}$ allele were used for backcrossings to C57BL/6NCrl, 129S6/SvEvTac, or CD1 and further analyses were carried out with Fltp2VAneo/+ mice backcrossed several generations in C57BL/6NCrl, 129S6/SvEvTac, or CD1.

\section{Generation of the Dlg3:Venus mouse line}

The Dlg3-Venus mouse line was generated by electroporating a pCAG-Venus-Dlg3 construct into IDG3.2-F1 ES cells (C57BL/6J x 129S6/SvEvTac). Positive ES cells were aggregated with CD1 morulae and the resulting chimeras gave germ-line transmission of the Dlg3-Venus allele.

\section{Isolation of mouse tracheal epithelial lung cells and ALI culture}

Isolation of mouse tracheal epithelial cells (mTECs) and air liquid interface (ALI) culture was performed as described in Vladar and Brody (Vladar and Brody, 2013).

Mice were sacrificed and the trachea dissected out. The trachea was put into ice cold HamF12 medium supplemented with penicillin/streptomycin (P/S). Tissue surrounding the trachea was removed under the dissecting microscope in HamF12 medium. The trachea was digested in HamF12 medium supplemented with $1.5 \mathrm{mg} / \mathrm{ml}$ pronase (protease 14 ) at $4^{\circ} \mathrm{C}$ ON. On the next day the tube was put on ice, FBS (fetal bovine serum) was added to a concentration of $10 \%$ and the tube was inverted at least 12x. The tracheas were transferred into a new tube with HamF12 P/S 10\% FBS medium and again inverted 12x. Tracheas of the same genotype can be pooled and centrifuged for $10 \mathrm{~min}$ at $4{ }^{\circ} \mathrm{C}$ at $400 \times \mathrm{g} .200 \mu \mathrm{l} \mathrm{HamF} 12 \mathrm{P} / \mathrm{S}$ per trachea were supplemented with $0.5 \mathrm{mg} / \mathrm{ml}$ DNasel and $10 \mathrm{mg} / \mathrm{ml} \mathrm{BSA}$ (bovine serum albumin), the trachea were resuspended and incubated for $5 \mathrm{~min}$ on ice. Subsequently, the tubes were centrifuged for $5 \mathrm{~min}$ at $4^{\circ} \mathrm{C}$ at $400 \times \mathrm{g}$. The cells were resuspended in MTEC basic medium (DMEM F12 [\#21331-020; Invitro], 15 mM HEPES [1M stock], $3.6 \mathrm{mM}$ sodium bicarbonate, $4 \mathrm{mM}$ L-Glut [200 mM stock], P/S [100Glux stock], Fungizone [500x stock]) with 10\% FBS and incubated for $3-4 \mathrm{hr}$ at $37^{\circ} \mathrm{C}$. The supernatant was collected, centrifuged for $5 \mathrm{~min}$ at $400 \times \mathrm{g}$, and resuspended in 100-200 $\mu$ I MTEC plus medium (MTEC basic, $10 \mu \mathrm{g} / \mathrm{ml}$ insulin $(5 \mathrm{mg} / \mathrm{ml} \mathrm{stock}$ ), $5 \mu \mathrm{g} / \mathrm{ml}$ transferrin ( $5 \mathrm{mg} / \mathrm{ml}$ stock), $0.1 \mu \mathrm{g} / \mathrm{ml}$ cholera toxin $(100 \mu \mathrm{g} / \mathrm{ml} \mathrm{stock}), 25 \mathrm{ng} / \mathrm{ml} \mathrm{EGF} \mathrm{(} 25 \mu \mathrm{g} / \mathrm{ml} \mathrm{stock})$, $30 \mu \mathrm{g} / \mathrm{ml} \mathrm{BPE}, 5 \% \mathrm{FBS}, 0.01 \mu \mathrm{M} \mathrm{RA}$ ) for counting. 75.000 cells per $\mathrm{cm}^{2}$ were seeded on a transwell filter in MTEC plus medium. The medium was changed every 2 days. When the cells were confluent the MTEC culture was changed to an ALI culture by removing the medium on top of the filter and providing only medium supply from the bottom.

\section{Immunoprecipitation}

For the interaction analysis, we used HEK293T cells transfected with Fltp-TAP-TAG and as a control HEK293T cells only transfected with TAP-TAG. 
The cells were rinsed with warm PBS and lysed with $1 \mathrm{ml}$ ice cold lysis buffer $(50 \mathrm{mM}$ Tris/ $/ \mathrm{HCl}$, $\mathrm{pH}$ 7.4, $150 \mathrm{mM}$ sodium chloride, 2 mM EDTA, pH 8, 1\% Nonidet P-40, filtrate sterile) per $10 \mathrm{~cm}$ dish on ice. Cells were spinned down for $10 \mathrm{~min}$ at $10000 \times \mathrm{g}$ at $4^{\circ} \mathrm{C}$. Lysates were cleaned by filtration through syringe filters (MILLEX GP, $0.22 \mu \mathrm{m}$, Millipore). $1 \mathrm{mg}$ of the filtered lysate was incubated with $50 \mu \mathrm{l}$ Strep-Tactin superflow resin (IBA) in $1 \mathrm{ml}$ of lysis buffer for $1 \mathrm{hr}$ at $4^{\circ} \mathrm{C}$ in an overhead tumbler. The protein-Strep-Tactin solution was spinned down for $30 \mathrm{~s}$ at $7000 \times g$ and transferred to microspin columns (GE-Healthcare). The supernatant was spinned down for $5 \mathrm{~s}$ at $100 \times \mathrm{g}$, washed three times with $500 \mu \mathrm{l}$ TBST $(100 \mathrm{mM}$ Tris/ $\mathrm{HCl}, \mathrm{pH} 7.4,1.5 \mathrm{M}$ sodium chloride, 1.0\% Tween 20 ), and centrifuged for $5 \mathrm{~s}, 100 \times \mathrm{g}$. The protein was eluted by $500 \mu \mathrm{l}$ desthiobiotin elution buffer (IBA) and incubated for 10 min while mixing the resin for several times. The elute can now be used for western blot analysis.

\section{Immunohistochemistry on cryosections and whole-mount immunohistochemistry}

Dissected tissues were fixed in $4 \%$ paraformaldehyde (PFA) and cryoprotected by incubation in a sucrose gradient for at least $1 \mathrm{hr}$ each (5\%, 15\%, 30\%). Tissues were frozen in OCT (optimal cutting temperature) after which immunohistochemical staining was carried out on 8- to 12- $\mu$ mthick sections, mounted on glass slides. Briefly, sections were rehydrated in PBS, permeabilised for 10 min in $0.1 \mathrm{M}$ glycine/0.1\% Triton X-100 in PBS, and blocked for $1 \mathrm{hr}$ in 5\% donkey serum/PBS-Tween 0.1\% (PBS-T). Finally, the sections were incubated with the primary antibody in blocking solution $\mathrm{ON}$ at $4^{\circ} \mathrm{C}$. The slides were washed with PBS-T, incubated with the secondary antibody in PBS for $2 \mathrm{hr}$ at RT, washed with PBS, incubated with DAPI, and finally mounted with ProLong Gold antifade reagent (P36930; Invitrogen). Whole-mount immunohistochemistry was performed as previously described (Nakaya et al., 2005). Briefly, embryos were isolated, fixed for $20 \mathrm{~min}$ in 2\% PFA in PBS, and then permeabilized in $0.1 \%$ Triton $\mathrm{X}-100$ in $0.1 \mathrm{M}$ glycine $\mathrm{pH}$ 8.0. After blocking in $10 \%$ FCS, $3 \%$ goat serum, $0.1 \%$ BSA, $0.1 \%$ Tween 20 for $2 \mathrm{hr}$, embryos were incubated with the primary antibody $\mathrm{ON}$ at $4^{\circ} \mathrm{C}$ in blocking solution. After several washes in PBS-T, embryos were incubated with secondary antibodies in blocking solution for $3 \mathrm{hr}$. During the final washes with PBS-T, embryos were stained with DAPI, transferred into $40 \%$ glycerol, and embedded between two coverslips using $120 \mu \mathrm{m}$ Secure-Seal spacers (S24737; Invitrogen) and ProLong Gold antifade reagent.

\section{Immunohistochemistry on inner ears}

Whole-mount inner ears were isolated, fixed for $20 \mathrm{~min}$ in 4\% PFA in PBS, then the cochlea was dissected out, permeabilized in $0.1 \%$ Triton X-100 in $0.1 \mathrm{M}$ glycine $\mathrm{pH}$ 8.0. After blocking in $10 \%$ FCS, $3 \%$ goat serum, $0.1 \%$ BSA, $0.1 \%$ Tween 20 for $25 \mathrm{~min}$, ears were incubated with the primary antibody $\mathrm{ON}$ at $4{ }^{\circ} \mathrm{C}$ in blocking solution. After several washes in PBS-T, embryos were incubated with secondary antibodies in blocking solution for at least $3 \mathrm{hr}$. During the final washes with PBS-T, embryos were stained with DAPI. Alternatively ears were fixed/permeabilized for $10 \mathrm{~min}$ in $0.25 \%$ glutaraldehyde, 3.7\% PFA, 3.7\% sucrose, and 0.1\% Triton X-100 in PHEM buffer (60 mM Pipes, 25 mM Hepes, $5 \mathrm{mM}$ EGTA, $1 \mathrm{mM} \mathrm{MgCl}$ ), the cochlea was dissected out and again fixed/permeabilized for $10 \mathrm{~min}$ in the same solution as above. After PBS washes blocking as described above was carried out. Finally, ears were embedded in a spacer between two cover slips.

\section{Immunohistochemistry on ALI cultures}

Antibody staining of ALI cultures was performed by cutting out, washing (PBS), and fixing (ice cold $\mathrm{MeOH}, 4 \% \mathrm{PFA}$, or fixed/permeabilised in $0.25 \%$ glutaraldehyde, $3.7 \%$ PFA, $3.7 \%$ sucrose, and $0.1 \%$ Triton X-100 in PHEM buffer depending on the primary antibody used) the Transwell membrane. Subsequently, membranes were treated as described above. Finally, membranes were embedded in a spacer between two cover slips.

\section{Genotyping of mouse lines}

Genotyping of the Fltp ${ }^{z \mathrm{~V}}$ mouse line (after Cre-mediated excision of the neomycin selection cassette) was performed using the forward primer 566 and 418 as well as the reverse primer 565 . With an annealing temperature of $57^{\circ} \mathrm{C}$ and 35 cycles, this PCR amplified a WT product of $317 \mathrm{bp}$ and a targeted of $387 \mathrm{bp}$.

For genotyping of the Dlg3:Venus mouse line, the forward primer 180 and the reverse primer 181 were used. A PCR performed with an annealing of $60^{\circ} \mathrm{C}$ and 32 cycles amplifies a product of $312 \mathrm{bp}$. 
Genotyping of Dlg3 mouse line was performed using the forward primer 534 and the reverse primer 535 as well as the reverse primer 536 . Amplification of 33 cycles with an annealing of $58^{\circ} \mathrm{C}$ yielded a WT product of $535 \mathrm{bp}$ and a $215 \mathrm{bp}$ product for the targeted allele. The gene trap clone P038A02 (R1 on a pure 129Sv6 genetic background) was obtained from the German Gene Trap Consortium. Dlg3tm1Grnt/Y male and Dlg3tm1Grnt/+ female mice on a C57Bl/6 background were genotyped as previously described (Cuthbert et al., 2007).

For genotyping of the Fltp ${ }^{\mathrm{zV}}$; Celsr 1 Crsh mouse line, we first performed a PCR with the forward primer 779 and the reverse primer 780 resulting in a $321 \mathrm{bp}$ band. Next, we purified the PCR product via the PCR purification kit and sequenced the product in both directions. The adenine of the WT sequence was replaced by a guanine in the mutated sequence. For the Fltp ${ }^{\mathrm{ZV}}$ genotyping, we used the protocol described above.

\section{Isolation of embryos and organs}

Dissections of embryos and organs were carried out according to Nagy and Behringer ('Manipulating the mouse embryo: a laboratory manual'). Embryos were staged according to Downs and Davies (Downs and Davies, 1993).

\section{Tissue clearing with BABB}

Tissues were dehydrated through a methanol/ $\mathrm{H}_{2} \mathrm{O}$ series: $2 \mathrm{hr}$ in $25 \%$ methanol/ $/ \mathrm{H}_{2} \mathrm{O}, 2 \mathrm{hr}$ in $50 \%$ methanol/ $/ \mathrm{H}_{2} \mathrm{O}, 2 \mathrm{hr}$ in $75 \%$ methanol/ $\mathrm{H}_{2} \mathrm{O}$, and $\mathrm{ON}$ in $100 \%$ methanol. Finally the tissue was transferred to $\mathrm{BABB}$ for clearing.

\section{Whole-mount in situ hybridization}

Whole-mount in situ hybridization was performed as previously described and Fltp mRNA was transcribed from a sequence-verified cDNA clone (Tamplin et al., 2008).

\section{Paraffin sections}

After in situ hybridization or lacZ staining, the embryos were dehydrated via a methanol series $(25 \%$, $50 \%, 75 \%, 2 \times 100 \%$ ) for 10 min per step. To clear the embryos, we incubated $2 \times$ in xylol for 5-10 min (depending on the thickness of the specimen). For tissue penetration of the paraffin, the specimen was left in paraffin at $65^{\circ} \mathrm{C} \mathrm{ON}$. On the next day, they were transferred into fresh paraffin and incubated at $65^{\circ} \mathrm{C} \mathrm{ON}$. The embryos were orientated and embedded into a mold. The cooled down paraffin blocks were mounted onto a grid and sectioned on a microtome. The sections were mounted on glass slides, dried $\mathrm{ON}$ at $37^{\circ} \mathrm{C}$, dewaxed in xylol $(2 \times 15 \mathrm{~min})$, fixed with mounting medium, and covered with a cover slip. After one night at $4^{\circ} \mathrm{C}$, the sections were ready for microscopical analyses.

\section{Histological staining of paraffin sections using Nuclear Fast Red (NFR)}

First, paraffin sections on glass slides were dewaxed twice for $15 \mathrm{~min}$ in xylene. An alcohol row $100 \%$, $90 \%, 80 \%, 70 \%, 1$ min each) for rehydration followed and ended in $\mathrm{H}_{2} \mathrm{O}$. Afterward, the slides were dipped into NFR for $1 \mathrm{~min}$ and thoroughly washed with dist. $\mathrm{H}_{2} \mathrm{O}$. Then, a dehydration step followed within an afferent alcohol row $(70-100 \%$, see above). Finally, the slides were incubated twice in xylene for 15-30 min and once in Roti-Histol for 15-30 min. After incubation in Roti-Histol, slides were put on a paper towel, sprinkled with a few drops mounting medium, and covered with a cover slip.

\section{Statistical analysis}

Statistical significance was calculated using Prism (GraphPad Software). Circular statistics were done using MATLAB and R.

\section{Image acquisition and analysis}

Image acquisition was performed on a Leica DMI 6000 confocal microscope or on a Zeiss Lumar.V12 Stereo using an AxioCam MRc5 camera. Image analysis was performed with Leica LAS AF software, AxioVision (Zeiss), and Imaris 7.6.4 (Bitplane, Zürich, Switzerland).

\section{Oligonucleotides for cloning}

5' S Fltp FWD: 5'-NNNCTCGAGGAGCCCTTACGCACACTTAAG-3'

5' S Fltp REV: 5'-NNNTCTAGACGGGACATTAACTGCATCTTATCTGAGGTTG-3'

011: 5'-NNNACTAGTAGGTAAGTGTACCCAATTCGCCCTATAG-3' 

012: 5'-NNNGGATCCACGCGTTAAGATACATTGATGAGTTTGGAC-3'
013: 5'-NNNTCTAGAATGGTGAGCAAGGGCGAGGAGCTGTTC-3'
014: 5'-NNNACTAGTTTACTTGTACAGCTCGTCCATGCCGAGAG-3'
025: 5'-NNNGCGGCCGCGCCACCATGCCAGAGCCAGCG-3'
026: 5'-NNNTCTAGACTTAGCGCTGGTGTACTTGGTGATGG-3'
340: 5'-NNNGCGGCCGCGCCACCATGAACCTTGAAGCTCGAAAAACAAAG-3'
341: 5'-NNNGGCGCGCCTTTTTGACACCAGACCAACTGGTAATGGTAGC-3'
449: 5'-NNNGGCGCGCCAGTCAGGAAGTGGAAGAGAAGAACACAG-3'
450: 5'-NNNAAGCTTACTAGTGTGGTGGAGTGCCTGTCTACATGTG-3'
451: 5'-NNNAAGCTTCACGACAGTCAAAGCTGCAATAGAAC-3'
452: 5'-NNNGGATCCGGTAATTTGGCAATTATAGAACTCAGGC-3'
453: 5'-NNNCCGCGGAGCAGACTTAACTATGTTGGGGAAACAGC-3'
454: 5'-NNNGTCGACGCGGCCGCTGTTTACACTTGTTGCCTGGCAACTG-3'
455: 5'-NNNGTCGACGGTCCTAGTCTAGCTGAGGTCCAGATC-3'
456: 5'-NNNGGTACCATGCTGTGGGAGTCACTGACATTCTTG-3'

\section{Oligonucleotides for genotyping}

180: 5'-GTGAACCGCATCGAGCTGAAGG-3'

181: 5'-GAACTCCAGCAGGACCATGTG-3'

418: 5'-AGCCATACCACATTTGTAGAGG-3'

534: 5'-GGTCTCTGATGAAGCAGTGATTTTT-3'

535: 5'-TGATGACCCATAGACAGTAGGATCA-3'

536: 5'-CTAAAGCGCATGCTCCAGAC-3'

565: 5'-CAGCATGGCATAGATCTGGAC-3'

566: 5'-GAGGCTGACTGGGAACAATC-3'

779: 5'-ACAACCTTTGGGCTCTCG-3'

780: 5'-TATAGTCCCTCCGGACCTC-3'

\section{Acknowledgements}

We are extremely grateful to A Theis and B Vogel for excellent technical assistance and mouse work, Neil Copeland for generously providing plasmids and bacterial strains for homologous recombination in bacteria, and Ralf Kühn for IDG3.2 ES cells. Special thanks goes to Christiane Fuchs (ICB Consulting) for the circular statistics. We are particularly grateful to Jennifer Murdoch for providing Celsr $1^{\text {crsh }}$ animals. This work was supported by an Emmy-Noether Fellowship and the European Union with the ERC starting grant CiliaryDisease dedicated to HL. For financial support, HL thanks the Helmholtz Society, German Research Foundation, and German Center for Diabetes Research (DZD e.V.). This work was funded (in part) by the Helmholtz Alliance ICEMED_Imaging and Curing Environmental Metabolic Diseases, through the Initiative and Networking Fund of the Helmholtz Association.

\section{Additional information}

Funding

\begin{tabular}{lll} 
Funder & Grant reference number & Author \\
\hline Deutsche Forschungsgemeinschaft & Emmy-Noether Fellowship & Heiko Lickert \\
\hline European Research Council & ERC CiliaryDisease & Heiko Lickert
\end{tabular}

The funders had no role in study design, data collection and interpretation, or the decision to submit the work for publication.

Author contributions

MG, AB, Conception and design, Acquisition of data, Analysis and interpretation of data, Drafting or revising the article; IB, Designed the Fltp knock out construct, Conception and design; $\mathrm{SH}$, Helped to establish ALI culture, Contributed unpublished essential data or reagents; CVC, Drafting 
or revising the article, Contributed unpublished essential data or reagents; MA, AW, Helped to analyse SEM data; SGNG, Provided the Dlg3 knock out animals; HL, Conception and design, Analysis and interpretation of data, Drafting or revising the article, Contributed unpublished essential data or reagents

Ethics

Animal experimentation: Mouse keeping was done at the central facilities at HMGU in accordance with the German animal welfare legislation and acknowledged guidelines of the Society of Laboratory Animals (GV-SOLAS) and of the Federation of Laboratory Animal Science Associations (FELASA). Post-mortem examination of organs was not subject to regulatory authorization.

\section{References}

Bellaiche Y, Gho M, Kaltschmidt JA, Brand AH, Schweisguth F. 2001. Frizzled regulates localization of cell-fate determinants and mitotic spindle rotation during asymmetric cell division. Nature Cell Biology 3:50-57. doi: $10.1038 / 35050558$.

Bergstralh DT, Haack T, St Johnston D. 2013. Epithelial polarity and spindle orientation: intersecting pathways. Philosophical Transactions of the Royal Society of London Series B, Biological Sciences 368:20130291. doi: 10.1098/rstb.2013.0291.

Burtscher I, Lickert H. 2009. Foxa2 regulates polarity and epithelialization in the endoderm germ layer of the mouse embryo. Development 136:1029-1038. doi: 10.1242/dev.028415.

Collier S, Gubb D. 1997. Drosophila tissue polarity requires the cell-autonomous activity of the fuzzy gene, which encodes a novel transmembrane protein. Development 124:4029-4037.

Curtin JA, Quint E, Tsipouri V, Arkell RM, Cattanach B, Copp AJ, Henderson DJ, Spurr N, Stanier P, Fisher EM, Nolan PM, Steel KP, Brown SD, Gray IC, Murdoch JN. 2003. Mutation of Celsr1 disrupts planar polarity of inner ear hair cells and causes severe neural tube defects in the mouse. Current Biology 13:1129-1133. doi: 10.1016/ S0960-9822(03)00374-9.

Cuthbert PC, Stanford LE, Coba MP, Ainge JA, Fink AE, Opazo P, Delgado JY, Komiyama NH, O'Dell TJ, Grant SG. 2007. Synapse-associated protein 102/dlgh3 couples the NMDA receptor to specific plasticity pathways and learning strategies. The Journal of Neuroscience 27:2673-2682. doi: 10.1523/JNEUROSCI.4457-06.2007.

Downs KM, Davies T. 1993. Staging of gastrulating mouse embryos by morphological landmarks in the dissecting microscope. Development 118:1255-1266.

Ezan J, Lasvaux L, Gezer A, Novakovic A, May-Simera H, Belotti E, Lhoumeau AC, Birnbaumer L, Beer-Hammer S, Borg JP, Le Bivic A, Nürnberg B, Sans N, Montcouquiol M. 2013. Primary cilium migration depends on G-protein signalling control of subapical cytoskeleton. Nature Cell Biology 15:1107-1115. doi: 10.1038/ncb2819.

Ezan J, Montcouquiol M. 2013. Revisiting planar cell polarity in the inner ear. Seminars in Cell \& Developmental Biology 24:499-506. doi: 10.1016/j.semcdb.2013.03.012.

Gerdes JM, Davis EE, Katsanis N. 2009. The vertebrate primary cilium in development, homeostasis, and disease. Cell 137:32-45. doi: 10.1016/j.cell.2009.03.023.

Gray RS, Abitua PB, Wlodarczyk BJ, Szabo-Rogers HL, Blanchard O, Lee I, Weiss GS, Liu KJ, Marcotte EM, Wallingford JB, Finnell RH. 2009. The planar cell polarity effector Fuz is essential for targeted membrane trafficking, ciliogenesis and mouse embryonic development. Nature Cell Biology 11:1225-1232. doi: 10.1038/ ncb1966.

Hitz C, Wurst W, Kuhn R. 2007. Conditional brain-specific knockdown of MAPK using Cre/loxP regulated RNA interference. Nucleic Acids Research 35:e90. doi: 10.1093/nar/gkm475.

Jain R, Pan J, Driscoll JA, Wisner JW, Huang T, Gunsten SP, You Y, Brody SL. 2010. Temporal relationship between primary and motile ciliogenesis in airway epithelial cells. American Journal of Respiratory Cell and Molecular Biology 43:731-739. doi: 10.1165/rcmb.2009-03280C.

Johnston CA, Hirono K, Prehoda KE, Doe CQ. 2009. Identification of an Aurora-A/PinsLINKER/Dlg spindle orientation pathway using induced cell polarity in S2 cells. Cell 138:1150-1163. doi: 10.1016/j.cell. 2009.07.041.

Jones C, Chen P. 2008. Primary cilia in planar cell polarity regulation of the inner ear. Current Topics in Developmental Biology 85:197-224. doi: 10.1016/S0070-2153(08)00808-9.

Kinzel D, Boldt K, Davis EE, Burtscher I, Trumbach D, Diplas B, Attie-Bitach T, Wurst W, Katsanis N, Ueffing M, Lickert H. 2010. Pitchfork regulates primary cilia disassembly and left-right asymmetry. Developmental Cell 19:66-77. doi: 10.1016/j.devcel.2010.06.005.

Knoblich JA. 2008. Mechanisms of asymmetric stem cell division. Cell 132:583-597. doi: 10.1016/j. cell.2008.02.007.

Korinek WS, Copeland MJ, Chaudhuri A, Chant J. 2000. Molecular linkage underlying microtubule orientation toward cortical sites in yeast. Science 287:2257-2259. doi: 10.1016/j.cell.2008.02.007.

Kunda P, Baum B. 2009. The actin cytoskeleton in spindle assembly and positioning. Trends in Cell Biology 19:174-179. doi: 10.1016/j.tcb.2009.01.006.

Lange A, Gegg M, Burtscher I, Bengel D, Kremmer E, Lickert H. 2012. Fltp(T2AiCre): a new knock-in mouse line for conditional gene targeting in distinct mono- and multiciliated tissues. Differentiation; Research in Biological Diversity 83:S105-S113. doi: 10.1016/j.diff.2011.11.003. 
Lee L, Tirnauer JS, Li J, Schuyler SC, Liu JY, Pellman D. 2000. Positioning of the mitotic spindle by a corticalmicrotubule capture mechanism. Science 287:2260-2262. doi: 10.1126/science.287.5461.2260.

Liao WP, Uetzmann L, Burtscher I, Lickert H. 2009. Generation of a mouse line expressing Sox17-driven Cre recombinase with specific activity in arteries. Genesis 47:476-483. doi: 10.1002/dvg.20520.

Lickert H, Van Campenhout CA. 2012. Evolution of the discs large gene family provides new insights into the establishment of apical epithelial polarity and the etiology of mental retardation. Communicative \& Integrative Biology 5:287-290. doi: 10.4161/cib.19792.

Liu $P$, Jenkins NA, Copeland NG. 2003. A highly efficient recombineering-based method for generating conditional knockout mutations. Genome Research 13:476-484. doi: 10.1101/gr.749203.

McClintock TS, Glasser CE, Bose SC, Bergman DA. 2008. Tissue expression patterns identify mouse cilia genes. Physiological Genomics 32:198-206. doi: 10.1152/physiolgenomics.00128.2007.

McKenzie E, Krupin A, Kelley MW. 2004. Cellular growth and rearrangement during the development of the mammalian organ of Corti. Developmental Dynamics 229:802-812. doi: 10.1002/dvdy.10500.

Metzger RJ, Klein OD, Martin GR, Krasnow MA. 2008. The branching programme of mouse lung development. Nature 453:745-750. doi: 10.1038/nature07005.

Montcouquiol M, Rachel RA, Lanford PJ, Copeland NG, Jenkins NA, Kelley MW. 2003. Identification of Vangl2 and Scrb1 as planar polarity genes in mammals. Nature 423:173-177. doi: 10.1038/nature01618.

Nakaya MA, Biris K, Tsukiyama T, Jaime S, Rawls JA, Yamaguchi TP. 2005. Wnt3a links left-right determination with segmentation and anteroposterior axis elongation. Development 132:5425-5436. doi: 10.1242/dev.02149.

Park TJ, Haigo SL, Wallingford JB. 2006. Ciliogenesis defects in embryos lacking inturned or fuzzy function are associated with failure of planar cell polarity and Hedgehog signaling. Nature Genetics 38:303-311. doi: $10.1038 /$ ng1753.

Park TJ, Mitchell BJ, Abitua PB, Kintner C, Wallingford JB. 2008. Dishevelled controls apical docking and planar polarization of basal bodies in ciliated epithelial cells. Nature Genetics 40:871-879. doi: 10.1038/ng.104.

Peng Y, Axelrod JD. 2012. Asymmetric protein localization in planar cell polarity: mechanisms, puzzles, and challenges. Current Topics in Developmental Biology 101:33-53. doi: 10.1016/B978-0-12-394592-1.00002-8.

Rodriguez OC, Schaefer AW, Mandato CA, Forscher P, Bement WM, Waterman-Storer CM. 2003. Conserved microtubule-actin interactions in cell movement and morphogenesis. Nature Cell Biology 5:599-609. doi: 10.1038/ncb0703-599.

Seifert JR, Mlodzik M. 2007. Frizzled/PCP signalling: a conserved mechanism regulating cell polarity and directed motility. Nature Reviews Genetics 8:126-138. doi: 10.1038/nrg2042.

Shima Y, Copeland NG, Gilbert DJ, Jenkins NA, Chisaka O, Takeichi M, Uemura T. 2002. Differential expression of the seven-pass transmembrane cadherin genes Celsr1-3 and distribution of the Celsr2 protein during mouse development. Developmental Dynamics 223:321-332. doi: 10.1002/dvdy.10054.

Siller KH, Doe CQ. 2008. Lis1/dynactin regulates metaphase spindle orientation in Drosophila neuroblasts. Developmental Biology 319:1-9. doi: 10.1016/j.ydbio.2008.03.018.

Soriano P. 1999. Generalized lacZ expression with the ROSA26 Cre reporter strain. Nature Genetics 21:70-71. doi: $10.1038 / 5007$

Storm van's Gravesande K, Omran H. 2005. Primary ciliary dyskinesia: clinical presentation, diagnosis and genetics. Annals of Medicine 37:439-449. doi: 10.1038/5007.

Tamplin OJ, Kinzel D, Cox BJ, Bell CE, Rossant J, Lickert H. 2008. Microarray analysis of Foxa2 mutant mouse embryos reveals novel gene expression and inductive roles for the gastrula organizer and its derivatives. BMC Genomics 9:511. doi: 10.1186/1471-2164-9-511.

Tarchini B, Jolicoeur C, Cayouette M. 2013. A molecular blueprint at the apical surface establishes planar asymmetry in cochlear hair cells. Developmental Cell 27:88-102. doi: 10.1016/j.devcel.2013.09.011.

Van Campenhout CA, Eitelhuber A, Gloeckner CJ, Giallonardo P, Gegg M, Oller H, Grant SG, Krappmann D, Ueffing M, Lickert H. 2011. Dlg3 trafficking and apical tight junction formation is regulated by nedd4 and nedd4-2 e3 ubiquitin ligases. Developmental Cell 21:479-491. doi: 10.1016/j.devcel.2011.08.003.

Vladar EK, Bayly RD, Sangoram AM, Scott MP, Axelrod JD. 2012. Microtubules enable the planar cell polarity of airway cilia. Current Biology 22:2203-2212. doi: 10.1016/j.cub.2012.09.046.

Vladar EK, Brody SL. 2013. Analysis of ciliogenesis in primary culture mouse tracheal epithelial cells. Methods Enzymology 525:285-309. doi: 10.1016/B978-0-12-397944-5.00014-6.

Vladar EK, Stearns T. 2007. Molecular characterization of centriole assembly in ciliated epithelial cells. The Journal of Cell Biology 178:31-42. doi: 10.1083/jcb.200703064.

Wallingford JB. 2012. Planar cell polarity and the developmental control of cell behavior in vertebrate embryos. Annual Review of Cell and Developmental Biology 28:627-653. doi: 10.1146/annurev-cellbio092910-154208.

Wang J, Mark S, Zhang X, Qian D, Yoo SJ, Radde-Gallwitz K, Zhang Y, Lin X, Collazo A, Wynshaw-Boris A, Chen P. 2005. Regulation of polarized extension and planar cell polarity in the cochlea by the vertebrate PCP pathway. Nature Genetics 37:980-985. doi: 10.1038/ng1622.

Wang Y, Guo N, Nathans J. 2006. The role of Frizzled3 and Frizzled6 in neural tube closure and in the planar polarity of inner-ear sensory hair cells. The Journal of Neuroscience 26:2147-2156. doi: 10.1523/ JNEUROSCI.4698-05.2005.

Wang Y, Nathans J. 2007. Tissue/planar cell polarity in vertebrates: new insights and new questions. Development 134:647-658. doi: 10.1242/dev.02772.

Weedon MN, Cebola I, Patch AM, Flanagan SE, De Franco E, Caswell R, Rodriguez-Segui SA, Shaw-Smith C, Cho $\mathrm{CH}$, Lango Allen H, Houghton JA, Roth CL, Chen R, Hussain K, Marsh P, Vallier L, Murray A, International 
Pancreatic Agenesis Consortium, Ellard S, Ferrer J, Hattersley AT. 2014. Recessive mutations in a distal PTF1A enhancer cause isolated pancreatic agenesis. Nature Genetics 46:61-64. doi: 10.1038/ng.2826.

Yates LL, Schnatwinkel C, Murdoch JN, Bogani D, Formstone CJ, Townsend S, Greenfield A, Niswander LA, Dean $\mathrm{CH}$. 2010. The PCP genes Celsr1 and Vangl2 are required for normal lung branching morphogenesis. Human Molecular Genetics 19:2251-2267. doi: 10.1093/hmg/ddq104.

You Y, Richer EJ, Huang T, Brody SL. 2002. Growth and differentiation of mouse tracheal epithelial cells: selection of a proliferative population. American Journal of Physiology Lung Cellular and Molecular Physiology 283:L1315-L1321. 